\title{
Exact solution of the Bose-Hubbard model on the Bethe lattice
}

\author{
Guilhem Semerjian, ${ }^{1}$ Marco Tarzia, ${ }^{2}$ and Francesco Zamponi ${ }^{1}$ \\ ${ }^{1}$ LPTENS, Unité Mixte de Recherche (UMR 8549) du CNRS et de l'ENS, \\ associée à l'UPMC Univ Paris 06, 24 Rue Lhomond, 75231 Paris Cedex 05, France. \\ ${ }^{2}$ Laboratoire de Physique Théorique de la Matière Condensée, Université Pierre et Marie Curie-Paris 6 , \\ UMR CNRS 7600, 4 place Jussieu, 75252 Paris Cedex 05, France.
}

\begin{abstract}
The exact solution of a quantum Bethe lattice model in the thermodynamic limit amounts to solve a functional self-consistent equation. In this paper we obtain this equation for the BoseHubbard model on the Bethe lattice, under two equivalent forms. The first one, based on a coherent state path integral, leads in the large connectivity limit to the mean field treatment of Fisher et al. [Phys. Rev. B 40, 546 (1989)] at the leading order, and to the bosonic Dynamical Mean Field Theory as a first correction, as recently derived by Byczuk and Vollhardt [Phys. Rev. B 77, 235106 (2008)]. We obtain an alternative form of the equation using the occupation number representation, which can be easily solved with an arbitrary numerical precision, for any finite connectivity. We thus compute the transition line between the superfluid and Mott insulator phases of the model, along with thermodynamic observables and the space and imaginary time dependence of correlation functions. The finite connectivity of the Bethe lattice induces a richer physical content with respect to its infinitely connected counterpart: a notion of distance between sites of the lattice is preserved, and the bosons are still weakly mobile in the Mott insulator phase. The Bethe lattice construction can be viewed as an approximation to the finite dimensional version of the model. We show indeed a quantitatively reasonable agreement between our predictions and the results of Quantum Monte Carlo simulations in two and three dimensions.
\end{abstract}

\section{INTRODUCTION}

The bosonic version of the Hubbard model was introduced for the first time in the seminal paper [1]. It describes a system of bosons hopping between neighboring sites of a lattice, and subjected to a local repulsive interaction disfavouring the multiple occupancy of a site. The competition between these two effects leads to a quantum phase transition [2] at zero temperature: when the hopping term dominates the groundstate is a Bose-Einstein Condensate (BEC) delocalized over the lattice, also known as a superfluid (SF) phase. On the contrary for large local repulsion it becomes energetically favorable to form a commensurate state where the average number of bosons per site is fixed to an integer value. This incompressible phase is called a Mott insulator (MI).

The introduction of this model was motivated by experimental results on Helium adsorbed on disordered substrates. The recent progresses of the experimental techniques for the manipulation of cold atoms, and in particular the possibility of devising optical lattices loaded with cold gases, revivified the interest for the Bose-Hubbard model. Following the proposal made in [3], the experimental observation of this Mott transition was first achieved in [4]. We refer the reader to [5] for a review of such experiments bridging a gap between atomic and condensed-matter physics.

From a more theoretical point of view the Bose-Hubbard model has been studied with various techniques, notably mean-field approximations $[1,6]$, perturbative expansions in the hopping strength [7-9], Random Phase Approximations (RPA) [10-14], and Quantum Monte Carlo simulations [15-20]. The mean-field approach of [1] yields a qualitatively correct prediction of the phase diagram of the model. However its description of the Mott insulator is oversimplified, the hopping being completely neglected in this phase at the mean-field level. As often the assumptions underlying the mean-field approximation become valid when the connectivity of each site (the number of its neighbors) is very large, either of the order of the system size itself (in which case a rigorous treatment of the model is possible $[21,22]$ ), or in the limit of large dimensionality. The latter case was recently investigated in $[23,24]$ where a bosonic version of the Dynamic Mean-Field Theory [25] was developed, including first order corrections in the inverse of the spatial dimension.

In this paper we follow a related but slightly different road to improve on the mean-field treatment of [1], by treating the Bose-Hubbard model at the level of the Bethe approximation, i.e. by solving it on a Bethe lattice. We show that, in the thermodynamic limit, the computation of all observables amounts to solve a single self-consistent functional equation. By writing this equation in the coherent state basis one recovers the B-DMFT in the large connectivity limit $[23,24]$. Unfortunately, this equation cannot be analytically solved for finite connectivity, except in some special limits. Hence, we show how to rewrite it in a more convenient way using the occupation number basis; this opens the way to its resolution through an efficient numerical algorithm.

The Bethe approximation is the next step in a hierarchy of approximations for lattice systems known as the Cluster Variation Method [26]; it is in fact exact when the model considered is defined on a Bethe lattice, that is a graph which is locally a tree (no short loops can be closed on it). Bethe lattices appear in the DMFT derivations [23-25] as an 
intermediate step, before taking the limit of large connectivity. On the contrary in the present paper the connectivity of the Bethe lattice will be kept finite. From the point of view of the universality classes of critical exponents Bethe lattice models fall in the mean-field category, yet their finite connectivity makes them richer than the (fully-connected) mean-field version of [1]. In the latter any site is adjacent to all others, whereas on a Bethe lattice one has a well defined notion of distance between two sites, though it does not correspond to the usual Euclidean distance. Moreover the MI phase is non-trivial for the Bethe lattice version of the model, at variance with the mean-field picture. Though we only consider here the ordered version of the model a motivation for studying the Bethe lattice is the possibility that it exhibits a Bose Glass phase [1,27] in presence of disorder, which cannot be described at the simplest mean-field level. This hope is backed up by the relevance of the Bethe lattices for the localization problem [28].

The methodology we use has its roots in the extensive research effort which took place over the last decade in the community studying statistical mechanics of disordered systems. Under the name of cavity method a set of techniques has been developed to solve classical spin models on Bethe lattices (in the sense of sparse random graphs), with applications to spin glasses [29] and to random optimization problems arising from theoretical computer science [30]. An extensive presentation of this field can be found in the recent book [31]. The cavity method has been recently extended from classical to quantum spin models in presence of a transverse field [32-34]. We widen here the range of applicability of the method by including lattice boson models.

The rest of the paper is organized as follows. In Sec. II we define precisely the model studied and the physical observables of interest, and we recall the usual mean-field treatment and its qualitative physical predictions. Sec. III is an overview conveying the main ideas of the cavity method, starting for pedagogical reasons from the ferromagnetic Ising model before explaining its extension to quantum problems, and in particular its connection with the B-DMFT of $[23,24]$. Sec. IV contains the core of our contribution; we first present in Sec. IV A the main equations describing the Bose-Hubbard model on the Bethe lattice, and the principles of their numerical resolution. In Sec. IV B we present our predictions for several physical observables and compare the phase diagram we obtain with the Quantum Monte Carlo results in two [17] and three [18] dimensions, as well as with the B-DMFT prediction of [24] in three dimensions. For the convenience of the reader not interested in technical aspects we postpone the detailed derivation of the equations to Sec. V. Finally we present our conclusions and perspectives for future work in Sec. VI.

\section{DEFINITIONS AND REMINDER ON THE TRADITIONAL MEAN-FIELD APPROACH}

\section{A. Definition of the model and of the observables}

We shall consider the Bose-Hubbard model for spinless bosons on a graph of $N$ vertices (sites) defined by the following Hamiltonian,

$$
H=-J \sum_{\langle i, j\rangle}\left(a_{i}^{\dagger} a_{j}+a_{j}^{\dagger} a_{i}\right)+\frac{U}{2} \sum_{i=1}^{N} a_{i}^{\dagger} a_{i}^{\dagger} a_{i} a_{i}-\mu \sum_{i=1}^{N} a_{i}^{\dagger} a_{i}=H_{K}+H_{L}
$$

where the first sum runs over a subset of the $N(N-1) / 2$ possible edges (links) between pairs of sites, $J$ is the hopping amplitude between neighboring sites, $a_{i}$ (resp. $a_{i}^{\dagger}$ ) is the boson annihilation (resp. creation) operator on site $i, \mu$ is the chemical potential fixing the density of particles, and $U$ controls the strength of the on-site interaction between particles. It is convenient to separate the kinetic term $H_{K}$ (proportional to $J$ ) in the Hamiltonian, from the local term $H_{L}$ including the Hubbard interaction and the chemical potential term. Note that the kinetic energy defined in such a way will be negative: indeed, the discrete lattice version of the kinetic energy would be given by $H_{K}+J \sum_{i} c_{i} a_{i}^{\dagger} a_{i}$ where $c_{i}$ is the connectivity of site $i$.

Although the method we will discuss in this paper allows in principle to compute very general observables (such as multi-point imaginary time correlations), in the following we will be mainly interested in standard observables such as the mean density, kinetic and on-site energy, condensate fraction, and Green functions. For the sake of clarity we recall now their definitions. The partition function at temperature $T$ is defined by

$$
Z=\operatorname{Tr}\left[e^{-\beta H}\right]
$$

where $\beta=1 / T$ (we set $k_{B}=1$ ), the corresponding free energy is $F=-T \log Z$; the free-energy per site is $f=F / N$. The thermodynamic average of an operator $O$ is defined as

$$
\langle O\rangle=\frac{1}{Z} \operatorname{Tr}\left[O e^{-\beta H}\right] .
$$


In particular the average number of particles on site $i$ reads $\left\langle n_{i}\right\rangle=\left\langle a_{i}^{\dagger} a_{i}\right\rangle$ and the density of particles is $\rho=$ $N^{-1} \sum_{i}\left\langle n_{i}\right\rangle$; the kinetic (resp. local) energy per site is $e_{K}=\left\langle H_{K}\right\rangle / N$ (resp. $e_{L}=\left\langle H_{L}\right\rangle / N$ ). To define the order parameter for BEC, one possibility that is convenient for our purposes is to introduce a small perturbation to the Hamiltonian in the form $H_{\varepsilon}=H+\varepsilon \sum_{i}\left(a_{i}+a_{i}^{\dagger}\right)$ and define

$$
\langle a\rangle=\lim _{\varepsilon \rightarrow 0}\langle a\rangle_{\varepsilon},
$$

where $\langle\bullet\rangle_{\varepsilon}$ denotes the thermodynamic average in presence of the perturbation.

The imaginary-time evolution of an operator $O$ is given by $O(\tau)=e^{\tau H} O e^{-\tau H}$; we then define the two-times correlation functions as [35]

$$
\begin{aligned}
& G_{>}^{i}(\tau)=\left\langle a_{i}(\tau) a_{i}^{\dagger}(0)\right\rangle=\frac{1}{Z} \operatorname{Tr}\left[e^{-(\beta-\tau) H} a_{i} e^{-\tau H} a_{i}^{\dagger}\right], \\
& G_{<}^{i}(\tau)=\left\langle a_{i}^{\dagger}(0) a_{i}(\tau)\right\rangle=\frac{1}{Z} \operatorname{Tr}\left[e^{-(\beta+\tau) H} a_{i}^{\dagger} e^{\tau H} a_{i}\right] .
\end{aligned}
$$

The Green function is defined, for $-\beta / 2 \leq \tau \leq \beta / 2$, by

$$
G^{i}(\tau)=\theta(\tau) G_{>}^{i}(\tau)+\theta(-\tau) G_{<}^{i}(\tau)=\left\langle T a_{i}(\tau) a_{i}^{\dagger}(0)\right\rangle,
$$

where $T$ is the usual time-ordering operation, which should not be confused with the temperature. Note that the cyclic property of the trace implies that $G_{<}^{i}(\tau)=G_{>}^{i}(\tau+\beta)$, hence

$$
G^{i}(\tau)=\theta(\tau) G_{>}^{i}(\tau)+\theta(-\tau) G_{>}^{i}(\beta+\tau),
$$

and the knowledge of $G_{>}^{i}(\tau)$ for $0 \leq \tau \leq \beta$ is enough to determine the Green function.

The cavity method allows also the computation of spatial correlation functions, we shall in particular determine the one-particle density matrix $\rho_{i j}=\left\langle a_{i}^{\dagger} a_{j}\right\rangle$.

\section{B. Review of mean-field results}

The inexistence of an analytical solution of the model for finite dimensional lattices has triggered a large amount of research effort on numerical simulations [15-19], pertubative expansions [7, 8], or mean-field treatments [1,6] of the problem. Let us briefly recall the various points of view and methodology that the mean-field approach usually encompasses. It can first be seen as an approximation to finite-dimensional models. Maybe the most satisfactory way to perform this approximation is by means of a variational method. One introduces a simpler trial (Gutzwiller) Hamiltonian where the sites are decoupled,

$$
H_{0}=\sum_{i=1}^{N}\left[-\psi_{i} a_{i}^{\dagger}-\psi_{i}^{*} a_{i}+\frac{U}{2} a_{i}^{\dagger} a_{i}^{\dagger} a_{i} a_{i}-\mu a_{i}^{\dagger} a_{i}\right],
$$

the $\psi_{i}$ being here free parameters. Note that $H_{0}$ breaks the particle conservation symmetry. The free-energy at inverse temperature $\beta$ of the original model, $F(\beta)$, can be upper-bounded as $F \leq F_{0}+\langle H\rangle_{0}-\left\langle H_{0}\right\rangle_{0}$, where $F_{0}(\beta)$ is the free-energy of the trial Hamiltonian and $\langle\cdot\rangle_{0}$ denotes a thermal average with respect to the trial Hamiltonian. The best variational description is thus obtained by minimizing the upper bound with respect to the parameters $\psi_{i}$. Assuming that all sites of the graph have the same number $c$ of neighbors $(c=2 d$ for a $d$-dimensional hypercubic lattice), the bound is minimized by taking the same value $\psi$ on all sites, which can be chosen real without loss of generality. The free-energy per site can then be upper-bounded as $f(\beta) \leq f_{\text {var }}(\beta)$, with

$$
f_{\text {var }}(\beta)=\inf _{\psi}\left[\frac{1}{c J} \psi^{2}-\frac{1}{\beta} \ln \operatorname{Tr}\left[e^{\beta \mu a^{\dagger} a-\beta \frac{U}{2} a^{\dagger} a^{\dagger} a a+\beta \psi\left(a+a^{\dagger}\right)}\right]\right],
$$

where the trace is over the Hilbert space of a single site. The physical properties of this variational free-energy are well-known [1]: at all temperatures and chemical potentials there is a transition encountered upon increasing the hopping intensity $J$ from an "insulating" phase, characterized by $\langle a\rangle=0$, to a "superfluid" phase with $\langle a\rangle \neq 0$. At zero temperature this transition line draws a series of lobes in the $(J / U, \mu / U)$ plane, inside each lobe the particle density being constrained to a given integer. These Mott Insulator phases are thus incompressible. There exists 
alternative ways to obtain the estimation (9) for the free-energy per site of the Bose-Hubbard Hamiltonian. The usual mean-field approximation consists indeed in replacing $a_{i}^{\dagger} a_{j}$ with $\left\langle a_{i}^{\dagger}\right\rangle a_{j}+a_{i}^{\dagger}\left\langle a_{j}\right\rangle-\left\langle a_{i}^{\dagger}\right\rangle\left\langle a_{j}\right\rangle$ in the hopping term of the original Hamiltonian, neglecting correlations between neighboring sites. This transforms the Hamiltonian into the site-decoupled form (8), with $\psi_{i}$ given by a sum of $\left\langle a_{j}\right\rangle$ over the neighbors $j$ of $i$. These expectation values are then computed with respect to the decoupled Hamiltonian, the self-consistency equations leading finally to the same expression (9) of the free-energy per site as the variational approach; the latter has however the advantage of being better controlled, in the sense that it provides a rigorous bound on the true free-energy of the system. Finally, another reasoning yielding this mean-field result consists in devising a model which has exactly (9) as its free-energy per site in the thermodynamic limit, instead of taking it as an approximation for the finite-dimensional case. As could be expected this model corresponds to the fully-connected version of the Hamiltonian (1), with the sum in the hopping term running over all possible pairs of sites, with a coupling constant $J$ inversely proportional to the size $N$ of the system in order to have an extensive thermodynamic limit. It has been shown rigorously in [21, 22] that in the thermodynamic limit the free-energy of this fully-connected model converges indeed to (9).

The above described mean-field treatment has limitations both of a quantitative nature (the approximation cannot be expected to be very precise for small dimensions) and of a qualitative one. In particular the MI phase is rather trivial; as the infimum in (9) is reached in $\psi=0$, the hopping of particles is completely suppressed in this phase. This drawback is particularly severe in the case of the disordered Bose-Hubbard model, for which it forbids the description of the Bose Glass phase predicted in [1]. In order to cure this defect of the mean-field treatment one has to account in some way for the correlations between neighboring sites. Thinking in terms of classical spin models, the mean-field approximation is the lowest level of a hierarchy of descriptions (known as Cluster Variation Method [26], or Kikuchi approximations [36]) which treats exactly larger and larger regions of the interaction graph. In this work we shall deal with the Bose-Hubbard model at the next level of the hierarchy, known as the Bethe approximation, in which correlations between nearest neighbors are explicitly taken into account. In the same way as the simplest mean-field approximation was exact for the fully-connected model, the Bethe approximation is exact for a family of graphs, known as Bethe lattices. In these graphs each site interacts with a finite number $c$ of neighbors, say $2 d$ in order to mimic a $d$-dimensional hypercubic lattice, but the short loops present in the latter are discarded: Bethe lattices have a local tree structure, see Fig. 1 for a picture of the local appearance of a Bethe lattice of connectivity $c=4$. For at least two reasons it is however better not to picture a Bethe lattice as a finite regular tree (usually called Cayley tree in this context). Indeed a regular tree is strongly inhomogenous, a finite fraction of its "volume" being very close to its "surface", and only the center of the Cayley tree has the bulk properties one is interested in. Moreover in the case of frustrated models the boundary conditions imposed on the leaves of the Cayley tree have to be chosen with particular care. For these two reasons it has become customary in the community of statistical mechanics of disordered systems, following [29], to define a Bethe lattice as a random c-regular graph [37], that is a graph chosen uniformly at random from the set of the graphs on $N$ vertices where all sites have precisely $c$ neighbors. These Bethe lattices are locally tree-like, their loops have typically a length diverging with the size $N$ of the system, yet they do not have any boundary, all sites playing the same role (in the same way as periodic boundary conditions impose translation invariance on a finite cubic lattice). The absence of an underlying finite-dimensional structure gives them a mean-field character, but their finite connectivity leads to a richer content than the fully-connected models. The free-energy of Bethe lattice models is self-averaging with respect to their random character in the thermodynamic limit. In other words for large enough $N$ a single sample is a good representative of the ensemble average. The so-called cavity method has been developed to treat classical spin models defined on such random graphs, and found important applications for optimization problems of theoretical computer science [30].

\section{OVERVIEW OF THE QUANTUM CAVITY METHOD}

\section{A. The cavity method for ferromagnetic Ising models}

For the sake of pedagogy we shall begin our presentation by the cavity method treatment of the ferromagnetic Ising model on the Bethe lattice. We consider the following energy function,

$$
H\left(\sigma_{1}, \ldots, \sigma_{N}\right)=-J \sum_{\langle i, j\rangle} \sigma_{i} \sigma_{j}-h \sum_{i} \sigma_{i},
$$

where the classical degrees of freedom $\sigma_{i}$ can take the values \pm 1 , the first term describing ferromagnetic $(J>0)$ interactions between neighbors on a graph of $N$ vertices, the second one corresponding to an uniform magnetic field. We shall repeatedly use in the following the notation $\partial i$ for the set of vertices adjacent to a given vertex $i$, i.e. for the sites which interact with $i$, and $\partial i \backslash j$ for those vertices around $i$ distinct from $j$. The Gibbs-Boltzmann probability 


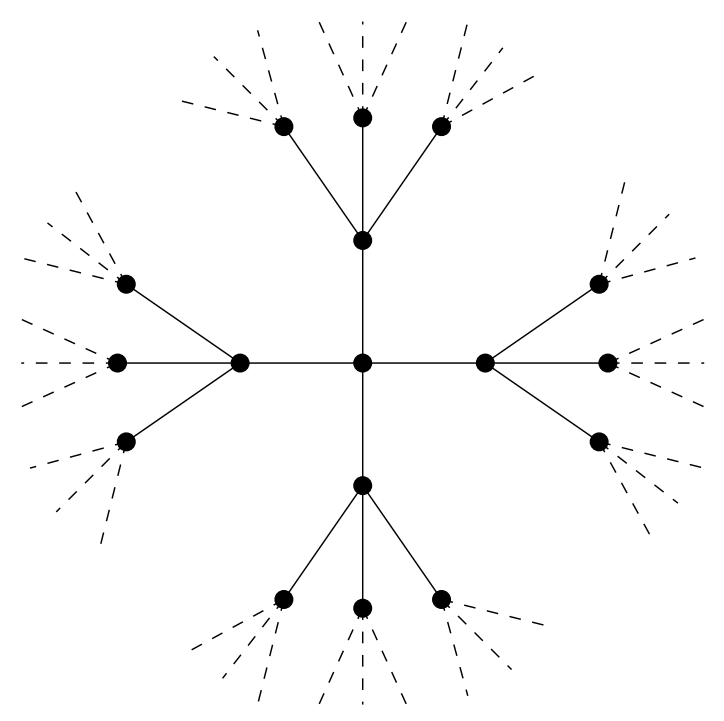

FIG. 1: A portion of a Bethe lattice of connectivity $c=4$, approximation of a square lattice.

measure is

$$
\eta\left(\sigma_{1}, \ldots, \sigma_{N}\right)=\frac{1}{Z} e^{-\beta H\left(\sigma_{1}, \ldots, \sigma_{N}\right)}
$$

with the partition function $Z$ ensuring its normalization. The goal is to compute the free-energy per site $f=$ $-(\ln Z) /(N \beta)$ and the local magnetizations $\left\langle\sigma_{i}\right\rangle$, the brackets denoting an average with respect to the law (11).

Let us first consider the case where the interaction graph is a finite tree. In this case the computation can be organized in a very simple way, taking benefit of the natural recursive structure of a tree. We define the quantity $Z_{i \rightarrow j}\left(\sigma_{i}\right)$, for two adjacent sites $i$ and $j$, as the partial partition function for the subtree rooted at $i$, excluding the branch directed towards $j$, with a fixed value of the spin variable on the site $i$. We also introduce $Z_{i}\left(\sigma_{i}\right)$, the partition function of the whole tree with a fixed value of $\sigma_{i}$. These quantities can be computed according to the following recursion rules, see Fig. 2 for an example,

$$
Z_{i \rightarrow j}\left(\sigma_{i}\right)=e^{\beta h \sigma_{i}} \prod_{k \in \partial i \backslash j}\left(\sum_{\sigma_{k}} Z_{k \rightarrow i}\left(\sigma_{k}\right) e^{\beta J \sigma_{i} \sigma_{k}}\right), \quad Z_{i}\left(\sigma_{i}\right)=e^{\beta h \sigma_{i}} \prod_{j \in \partial i}\left(\sum_{\sigma_{j}} Z_{j \rightarrow i}\left(\sigma_{j}\right) e^{\beta J \sigma_{i} \sigma_{j}}\right) .
$$

It will be useful for the following discussion to rewrite these equations in terms of normalized quantities which can be interpreted as probability laws for the random variable $\sigma_{i}$, namely $\eta_{i \rightarrow j}\left(\sigma_{i}\right)=Z_{i \rightarrow j}\left(\sigma_{i}\right) / \sum_{\sigma^{\prime}} Z_{i \rightarrow j}\left(\sigma^{\prime}\right)$ and $\eta_{i}\left(\sigma_{i}\right)=Z_{i}\left(\sigma_{i}\right) / \sum_{\sigma^{\prime}} Z_{i}\left(\sigma^{\prime}\right)$. The recursion equations read in these notations

$$
\eta_{i \rightarrow j}\left(\sigma_{i}\right)=\frac{1}{z_{i \rightarrow j}} e^{\beta h \sigma_{i}} \prod_{k \in \partial i \backslash j}\left(\sum_{\sigma_{k}} \eta_{k \rightarrow i}\left(\sigma_{k}\right) e^{\beta J \sigma_{i} \sigma_{k}}\right), \quad \eta_{i}\left(\sigma_{i}\right)=\frac{1}{z_{i}} e^{\beta h \sigma_{i}} \prod_{j \in \partial i}\left(\sum_{\sigma_{j}} \eta_{j \rightarrow i}\left(\sigma_{j}\right) e^{\beta J \sigma_{i} \sigma_{j}}\right),
$$

where $z_{i \rightarrow j}$ and $z_{i}$ are normalization constants. On a given tree the recursion equations on the $\eta_{i \rightarrow j}$ for all directed edges of the graph have a single solution, easily found by propagating the recursion from the leaves of the graph. Moreover the quantity $\eta_{i}\left(\sigma_{i}\right)$ is exactly the marginal probability law of the Gibbs-Boltzmann distribution (11), hence the local magnetizations can be computed as $\left\langle\sigma_{i}\right\rangle=\sum_{\sigma} \eta_{i}(\sigma) \sigma$.

The reasoning above was made under the assumption that the interaction graph was a tree. One can however look for a solution of the recursion equations (13) on any graph, even in the presence of loops. This approach is known as Belief Propagation in inference problems [38], and corresponds to the Bethe approximation of statistical mechanics [39]. The cavity method is a set of prescriptions to use these local recursion equations to predict the behavior of models defined on sparse random graphs, that are locally tree-like (the typical length of the loops diverging in the thermodynamic limit). In its simplest version (known as the replica symmetric one) one makes the assumption of the existence of a single pure state which implies that the effect of the loops does not spoil the local recursions (13). Their presence simply provides self-consistent boundary conditions and avoids the ill-defined behaviour due to the 


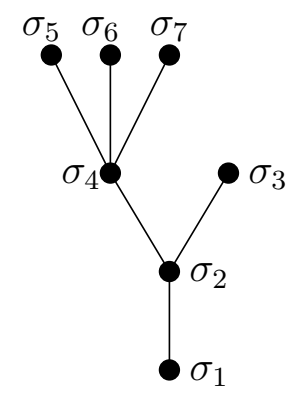

FIG. 2: Example of an Ising tree model on 7 vertices. The definition of $Z_{2 \rightarrow 1}$ and its recursive computation reads here: $Z_{2 \rightarrow 1}\left(\sigma_{2}\right)=\sum_{\sigma_{3}, \ldots, \sigma_{7}} e^{\beta h\left(\sigma_{2}+\sigma_{3}+\cdots+\sigma_{7}\right)+\beta J\left(\sigma_{2}\left(\sigma_{3}+\sigma_{4}\right)+\sigma_{4}\left(\sigma_{5}+\sigma_{6}+\sigma_{7}\right)\right)}=e^{\beta h \sigma_{2}} \sum_{\sigma_{3}, \sigma_{4}} Z_{3 \rightarrow 2}\left(\sigma_{3}\right) Z_{4 \rightarrow 2}\left(\sigma_{4}\right) e^{\beta J \sigma_{2}\left(\sigma_{3}+\sigma_{4}\right)}$.

dominant surface effects of trees. In the case of unfrustrated ferromagnetic models it has been shown rigorously [40] that this assumption is correct, the predictions of the cavity method being exact in the thermodynamic limit, both for the local magnetizations and for the free-energy per site. Let us be more explicit for the case of the Bethe lattice, where all vertices have the same connectivity $c$. The probability laws $\eta_{i \rightarrow j}$ are then the same on all edges; denoting $\eta_{\text {cav }}$ their common value, one finds the self-consistent equation

$$
\eta_{\mathrm{cav}}(\sigma)=\frac{1}{z_{\mathrm{cav}}} e^{\beta h \sigma} \sum_{\sigma_{1}, \ldots, \sigma_{c-1}} \eta_{\mathrm{cav}}\left(\sigma_{1}\right) \ldots \eta_{\mathrm{cav}}\left(\sigma_{c-1}\right) e^{\beta J \sigma\left(\sigma_{1}+\cdots+\sigma_{c-1}\right)},
$$

with $z_{\text {cav }}$ a normalization constant. A pictorial representation of this equation can be found in Fig. 3. The local magnetization is then computed as

$$
\langle\sigma\rangle=\sum_{\sigma} \eta(\sigma) \sigma, \quad \eta(\sigma)=\frac{1}{z} e^{\beta h \sigma} \sum_{\sigma_{1}, \ldots, \sigma_{c}} \eta_{\mathrm{cav}}\left(\sigma_{1}\right) \ldots \eta_{\mathrm{cav}}\left(\sigma_{c}\right) e^{\beta J \sigma\left(\sigma_{1}+\cdots+\sigma_{c}\right)},
$$

including the $c$ neighbors of a central site as represented in Fig. 4. The term cavity comes from the fact that in Eq. (14) one site has been removed from the neighborhood of the considered vertex. As the Ising variable $\sigma$ can only take two values, each of the probability distributions $\eta_{\text {cav }}$ and $\eta$ can be parametrized by a single real number, a cavity (resp. effective) magnetic field,

$$
\eta_{\mathrm{cav}}(\sigma)=\frac{e^{\beta h_{\mathrm{cav}} \sigma}}{2 \cosh \left(\beta h_{\mathrm{cav}}\right)}, \quad \eta(\sigma)=\frac{e^{\beta h_{\mathrm{eff}} \sigma}}{2 \cosh \left(\beta h_{\mathrm{eff}}\right)},
$$

solutions of

$$
h_{\text {cav }}=h+\frac{c-1}{\beta} \operatorname{arctanh}\left[\tanh (\beta J) \tanh \left(\beta h_{\text {cav }}\right)\right], \quad h_{\text {eff }}=h+\frac{c}{\beta} \operatorname{arctanh}\left[\tanh (\beta J) \tanh \left(\beta h_{\text {cav }}\right)\right] .
$$

thus making the resolution of (14),(15) extremely simple. In particular at zero external field $h=0$, one finds that a phase transition occurs at $\beta=\beta_{c}$, separating a high temperature paramagnetic phase $\left(h_{\text {cav }}=h_{\text {eff }}=0\right)$ from a low temperature ferromagnetic phase $\left(h_{\text {cav }}, h_{\text {eff }} \neq 0\right)$. The critical temperature is easily obtained linearizing the equation for $h_{\text {cav }}$ around $h_{\text {cav }}=0$ and is the solution of $(c-1) \tanh \left(\beta_{c} J\right)=1$.

It is worth noting that one can compute explicitly the spin-spin correlation function, which is given in the paramagnetic phase by $C_{i j}=\left\langle\sigma_{i} \sigma_{j}\right\rangle=[\tanh (\beta J)]^{d(i, j)}$, where $d(i, j)$ is the distance between sites $i$ and $j$, defined as the length of the shortest path connecting sites $i$ and $j$. The associated correlation length is $\xi=-\log [\tanh (\beta J)]$ which is finite at the transition point $\beta=\beta_{c}$. Nevertheless, the associated magnetic susceptibility $\chi=N^{-1} \sum_{i} \mathrm{~d}\left\langle\sigma_{i}\right\rangle / \mathrm{d} h$ diverges: one can show using the fluctuation-dissipation theorem that $\chi=\beta N^{-1} \sum_{i j} C_{i j}=\beta \sum_{d=0}^{\infty} \mathcal{N}_{d} C_{d}$, where $\mathcal{N}_{d}$ is the number of points at distance $d$ from a given reference point and scales as $(c-1)^{d}$ for large $d$. Therefore, if $C_{d}$ decays slower than $(c-1)^{-d}$, the corresponding susceptibility is divergent; this is indeed consistent with the equation for $\beta_{c}$ obtained above. Hence phase transitions on Bethe lattices are always associated to diverging susceptibilities and finite correlations lengths (see [41] for a discussion of this fact in the context of quantum spin models). For finite dimensional lattices, $\mathcal{N}_{d}$ grows as a power of $d$, and one needs a diverging correlation length to obtain a diverging susceptibility. 

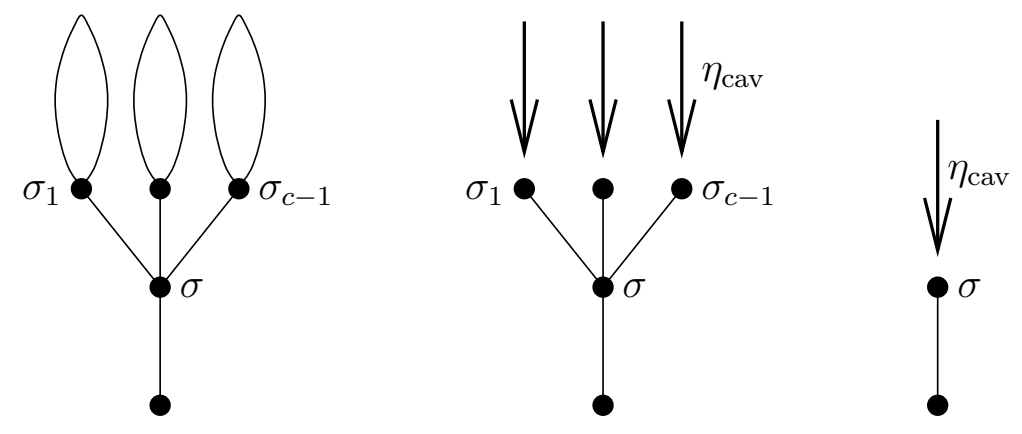

FIG. 3: Pictorial representation of Eq. (14). The bubbles on the first panel represent subtrees of the graph; their effect on the spins $\sigma_{1}, \ldots \sigma_{c-1}$ is summarized by $\eta_{\text {cav }}$, represented as a bold arrow in the second panel. Tracing over these $c-1$ spins leads to the third panel.
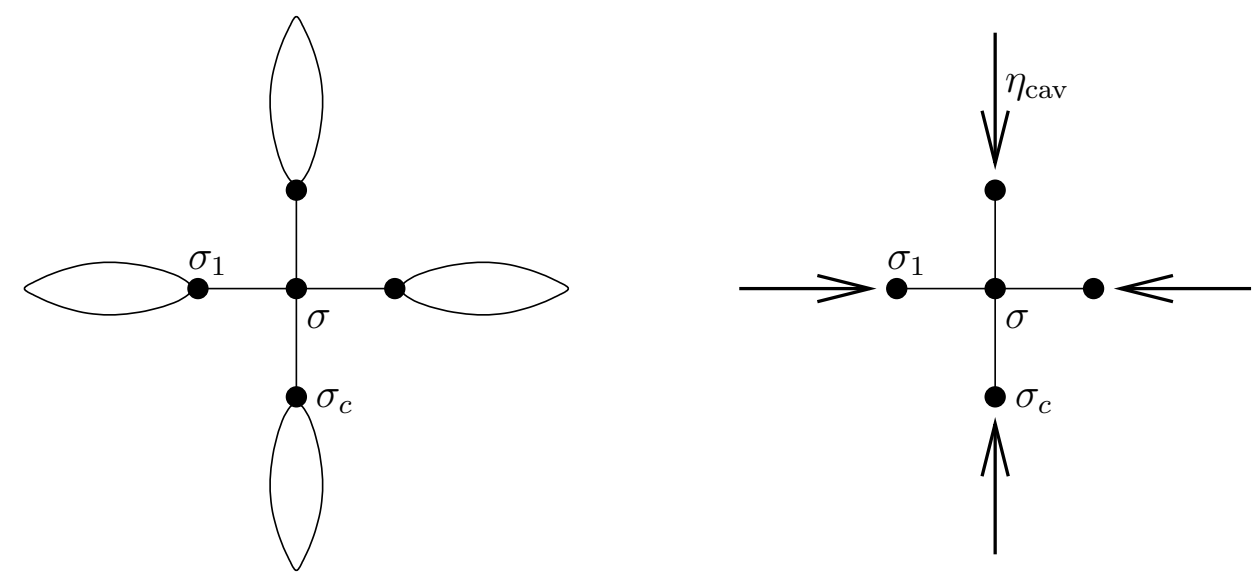

FIG. 4: Illustration of Eq. (15); the local magnetization of one site is computed by taking into account all the $c$ neighbors.

\section{B. Suzuki-Trotter formalism and quantum cavity method}

We have just seen how the cavity method deals with classical spin models on locally tree-like graphs. The extension of the method to quantum models is based on the Suzuki-Trotter formula. Generically speaking the resolution of a quantum model amounts to compute its partition function $Z=\operatorname{Tr}\left[e^{-\beta H}\right]$, where the Hamiltonian $H$ is now an operator, which can usually be split as $H=H_{1}+H_{2}$ with $H_{1,2}$ two operators which do not commute. For instance in the case of quantum spin $1 / 2$ models one can have an interaction term $H_{1}$ involving only the $z$ component of the spins, while $\mathrm{H}_{2}$ is a transverse field acting in a perpendicular direction. In the case of the Bose-Hubbard model this decomposition splits the Hamiltonian between the hopping term $H_{K}$ and the on-site potential energy $H_{L}$. The non-commutativity of $H_{1,2}$ can be cured with the application of the Suzuki-Trotter formula [42],

$$
Z=\lim _{N_{\mathrm{s}} \rightarrow \infty} \operatorname{Tr}\left[\left(e^{-\frac{\beta}{N_{\mathrm{s}}} H_{1}} e^{-\frac{\beta}{N_{\mathrm{s}}} H_{2}}\right)^{N_{\mathrm{s}}}\right]
$$

The computation then proceeds with the insertion of $N_{\mathrm{s}}$ representations of the identity between the $N_{\mathrm{s}}$ elements of this product. It is convenient to express the identity operator in a basis where one of the two operators is easily evaluated. In the case of quantum spin $1 / 2$ models one can for instance use the eigenvectors of the Pauli matrix in the $z$ direction; the quantum partition function is then turned into the partition function of a classical Ising spin model, where each quantum spin is replaced by a set of $N_{\mathrm{s}}$ classical spins, indexed by their position on the original graph and 
a supplementary "discrete imaginary time" coordinate (which becomes a continuous parameter in the $N_{\mathrm{s}} \rightarrow \infty$ limit). The important point is that the spatial structure of the graph of interactions is preserved in this construction, at the price of the introduction of imaginary time-dependent classical degrees of freedom. In particular, if the interactions of the quantum model are defined on a tree-like graph, the cavity method still applies to this extended classical model. This line of thought was first followed for quantum spin models in [32] (see also [33]) for a finite number $N_{\mathrm{s}}$ of Suzuki-Trotter slices, the continuous imaginary time limit was then taken in [34]. In this paper we shall extend this method to deal with lattice bosons models.

In this case the decomposition of the identity operator in the Suzuki-Trotter can be expressed using either coherent states or occupation numbers. The latter has the advantage of being discrete, and we shall use it in the rest of the paper. For the sake of clarity and in order to make contact with the recently proposed B-DMFT [23, 24] we discuss first the application of the cavity method within the coherent states representation in the rest of this subsection. Inserting such a decomposition of the identity for each of the $N$ sites at each of the $N_{\mathrm{s}}$ Suzuki-Trotter slices leads, in the continuous time limit $N_{\mathrm{s}} \rightarrow \infty$, to the coherent state path integral expression of the partition function of the Bose-Hubbard model [35]:

$$
\begin{aligned}
Z & =\int \prod_{i=1}^{N} D \boldsymbol{a}_{i} D \overline{\boldsymbol{a}}_{i} e^{-S} \\
S & =\int_{0}^{\beta} \mathrm{d} \tau\left[\sum_{i=1}^{N}\left(\bar{a}_{i}(\tau)\left(\partial_{\tau}-\mu\right) a_{i}(\tau)+\frac{U}{2}\left(\bar{a}_{i}(\tau) a_{i}(\tau)\right)^{2}\right)-J \sum_{\langle i, j\rangle}\left(\bar{a}_{i}(\tau) a_{j}(\tau)+\bar{a}_{j}(\tau) a_{i}(\tau)\right)\right]
\end{aligned}
$$

Here and in the following we use bold symbols to denote imaginary-time dependent quantities. $a_{i}(\tau)$ and $\bar{a}_{i}(\tau)$ are two (formally conjugate) complex numbers indexing the coherent state at site $i$ and imaginary time $\tau$, with $D \boldsymbol{a}_{i} D \overline{\boldsymbol{a}}_{i}$ the path-integral measure of this site. Following the same steps as in the study of the Ising model, the cavity method for a Bethe lattice of connectivity $c$ leads to the following self-consistency equation:

$$
\eta_{\mathrm{cav}}(\boldsymbol{a}, \overline{\boldsymbol{a}})=\frac{1}{z_{\mathrm{cav}}} w(\boldsymbol{a}, \overline{\boldsymbol{a}}) \int \prod_{i=1}^{c-1} D \boldsymbol{a}_{i} D \overline{\boldsymbol{a}}_{i} \eta_{\mathrm{cav}}\left(\boldsymbol{a}_{i}, \overline{\boldsymbol{a}}_{i}\right) \exp \left\{J \int_{0}^{\beta} \mathrm{d} \tau\left[\bar{a}(\tau) \sum_{i=1}^{c-1} a_{i}(\tau)+a(\tau) \sum_{i=1}^{c-1} \bar{a}_{i}(\tau)\right]\right\}
$$

with the on-site weight of the path $(\boldsymbol{a}, \overline{\boldsymbol{a}})$ given by

$$
w(\boldsymbol{a}, \overline{\boldsymbol{a}})=\exp \left[-\int_{0}^{\beta} \mathrm{d} \tau\left(\bar{a}(\tau)\left(\partial_{\tau}-\mu\right) a(\tau)+\frac{U}{2}(\bar{a}(\tau) a(\tau))^{2}\right)\right] .
$$

This self-consistent equation on $\eta_{\text {cav }}$ is formally similar to the corresponding Eq. (14) of the Ising model. It is however much more complicated: the Ising degree of freedom $\sigma$ could only take two distinct values, whereas $(\boldsymbol{a}, \overline{\boldsymbol{a}})$ belongs to a space of functions of the imaginary time $\tau$. Therefore $\eta_{\text {cav }}(\boldsymbol{a}, \overline{\boldsymbol{a}})$ is a functional measure whose representation is much more difficult; a complete parametrization requires the knowledge of all $n, m$-points correlations $\left\langle a\left(t_{1}\right) \cdots a\left(t_{n}\right) a^{\dagger}\left(s_{1}\right) \cdots a^{\dagger}\left(s_{m}\right)\right\rangle$. On the other hand, this is one of the most interesting features of the quantum cavity method: on-site quantum fluctuations are fully kept into account, without any truncation of higher order correlations.

Unfortunately, an exact solution of (21) can be easily obtained only in the case of free bosons $(U=0)$. $\eta_{\text {cav }}$ acquires in this case a Gaussian form, with averages and two-point functions which can be computed exactly and reproduce the results obtained by direct diagonalization of the adjacency matrix of the Bethe lattice [43].

\section{Large connectivity limit and the connection with B-DMFT}

In the interacting case $(U>0)$ a solution of $(21)$ can be looked for in the limit of large connectivity, and this is precisely the road followed by the B-DMFT studies [23, 24]. For completeness we shall explain in this subsection how to recover the B-DMFT formalism from Eq. (21), before turning in the next section to the occupation number basis which will allow to solve the model for any connectivity.

To state the large $c$ expansion let us rewrite (21) in a more convenient way, using the lighter Nambu notation $\Psi^{\dagger}(\tau)=(\bar{a}(\tau), a(\tau))$, and consequently $\Psi^{\dagger}=(\overline{\boldsymbol{a}}, \boldsymbol{a})$. Then we can rewrite (21) as

$$
\eta_{\text {cav }}(\boldsymbol{\Psi})=\frac{1}{z_{\text {cav }}} w(\boldsymbol{\Psi}) e^{(c-1) \Gamma(J \Psi)}, \quad \Gamma(\boldsymbol{\Phi})=\log \left\{\int D \boldsymbol{\Psi} \eta_{\text {cav }}(\boldsymbol{\Psi}) \exp \left[\int_{0}^{\beta} \mathrm{d} \tau \Psi^{\dagger}(\tau) \Phi(\tau)\right]\right\}
$$


$\Gamma(\boldsymbol{\Phi})$ is the generating functional of the connected correlation functions of $a$ and $\bar{a}$. It can be expanded as

$$
\Gamma(\boldsymbol{\Phi})=\int_{0}^{\beta} \mathrm{d} \tau\left\langle\Psi^{\dagger}\right\rangle \Phi(\tau)+\int_{0}^{\beta} \mathrm{d} \tau \mathrm{d} \tau^{\prime} \Phi^{\dagger}(\tau) \widehat{G}_{c}\left(\tau-\tau^{\prime}\right) \Phi\left(\tau^{\prime}\right)+O\left(\Phi^{3}\right)
$$

where the averages $\langle\cdot\rangle$ are with respect to $\eta_{\text {cav }}$, we have used the cyclic invariance in imaginary time, and $\widehat{G}_{c}\left(\tau-\tau^{\prime}\right)=$ $\left\langle\Psi(\tau) \Psi^{\dagger}\left(\tau^{\prime}\right)\right\rangle-\langle\Psi\rangle\left\langle\Psi^{\dagger}\right\rangle$ is the connected part of the two point correlator of $\boldsymbol{\Psi}$.

In the large connectivity limit, the superfluid-insulator transition happens for a critical value of $J=\mathcal{J} / c$, with a finite $\mathcal{J}$. This can be argued by looking at Eq. (9), where it is clear that the dependence on hopping and connectivity is only through $\mathcal{J}=J c$, which is the real control parameter. For large $c$ and $J=\mathcal{J} / c$, the cumulant expansion of $(c-1) \Gamma(\mathcal{J} \Psi / c)$ thus becomes a systematic expansion in powers of $1 / c$. By keeping only a finite number of terms in the cumulant expansion, we obtain an expression of $\eta_{\text {cav }}(\boldsymbol{\Psi})$ that is not Gaussian (because of the $U$ term in $w(\boldsymbol{\Psi})$ ); still, we can obtain closed equations for the cumulants by computing them self-consistently as averages over the non-Gaussian $\eta_{\text {cav }}$.

The leading order in $c$ gives, assuming without loss of generality that the average value of the order parameter is real, $\left\langle\Psi^{\dagger}\right\rangle=(\psi, \psi)$,

$$
\eta_{\text {cav }}(\Psi)=\frac{1}{z_{\text {cav }}} w(\Psi) e^{\int_{0}^{\beta} \mathrm{d} \tau \mathcal{J}\left\langle\Psi^{\dagger}\right\rangle \Psi(\tau)} \quad \Rightarrow \quad \psi=\frac{1}{z_{\text {cav }}} \int D \boldsymbol{a} D \overline{\boldsymbol{a}} a(0) e^{-\int_{0}^{\beta} \mathrm{d} \tau\left(\bar{a}(\tau)\left(\partial_{\tau}-\mu\right) a(\tau)+\frac{U}{2}(\bar{a}(\tau) a(\tau))^{2}-\mathcal{J} \psi(a(\tau)+\bar{a}(\tau))\right)}
$$

This last equation can be rewritten in the operator representation, which gives back the equation for $\psi$ corresponding to the minimization of the variational free-energy (9), up to a multiplicative constant in the definition of $\psi$. Note that a generalization of the discussion above and of Eq. (25) to the disordered case leads to the stochastic mean field theory devised in [44].

We will now show that the next-to-leading order in the cumulant expansion of (24) gives the B-DMFT equations recently derived in $[23,24]$. Note that the truncation at this two-point level was also used in the context of spin models in [32]. Plugging the expansion (24) in (23), we obtain to order $1 / c$ :

$$
\begin{aligned}
& \eta_{\text {cav }}(\Psi)=\frac{1}{z_{\text {cav }}} \exp \left[-S_{\text {loc }}\right], \\
& S_{\text {loc }}=\int_{0}^{\beta} \mathrm{d} \tau \mathrm{d} \tau^{\prime} \Psi^{\dagger}(\tau) \widehat{\mathcal{G}}^{-1}\left(\tau-\tau^{\prime}\right) \Psi\left(\tau^{\prime}\right)+\int_{0}^{\beta} \mathrm{d} \tau\left[\frac{U}{8}\left(\Psi^{\dagger}(\tau) \Psi(\tau)\right)^{2}-\mathcal{J} \frac{c-1}{c}\left\langle\Psi^{\dagger}\right\rangle \Psi(\tau)\right], \\
& \widehat{\mathcal{G}}^{-1}\left(\tau-\tau^{\prime}\right)=\frac{1}{2}\left(\begin{array}{cc}
\partial_{\tau}-\mu & 0 \\
0 & -\partial_{\tau}-\mu
\end{array}\right) \delta\left(\tau-\tau^{\prime}\right)-\frac{\mathcal{J}^{2}}{c} \widehat{G}_{c}\left(\tau-\tau^{\prime}\right) .
\end{aligned}
$$

Then, $\left\langle\Psi^{\dagger}\right\rangle$ and $\widehat{G}_{c}\left(\tau-\tau^{\prime}\right)=\left\langle\Psi(\tau) \Psi^{\dagger}\left(\tau^{\prime}\right)\right\rangle-\langle\Psi\rangle\left\langle\Psi^{\dagger}\right\rangle$ have to be computed self-consistently as averages with the local action $S_{\text {loc }}$. This set of equations correspond exactly to the B-DMFT of $[23,24]$ for the special case of a Bethe lattice.

Away from these two limits $(U=0$ and $c \rightarrow \infty)$ it seems difficult to obtain a solution of the cavity equation (21) as written in the coherent state basis. As a consequence we shall turn in the following to the representation number basis to apply the Suzuki-Trotter formula and thus obtain a more tractable equation for all values of $U$ and $c$.

\section{THE QUANTUM CAVITY METHOD IN THE OCCUPATION NUMBER BASIS}

\section{A. The equations and the procedure for their numerical resolution}

The insertion of a decomposition of the identity expressed in the occupation number basis in the Suzuki-Trotter formula (18) leads to an expression of the partition function of the Bose-Hubbard model as a sum over occupation number trajectories in imaginary time, $\left\{n_{i}(\tau)\right\}$. These trajectories are defined on an imaginary time interval of length $\beta$, with the periodicity condition $n_{i}(0)=n_{i}(\beta)$. The weight (action) of these trajectories has two origins: the local part of the Hamiltonian (1) yields a contribution of the form $\exp \left[-\int \mathrm{d} \tau V\left(n_{i}(\tau)\right)\right]$ for each of the sites, where $V(n)=U n(n-1) / 2-\mu n$ is the local energy term in the Hamiltonian. In addition the hopping term of the Hamiltonian imposes constraints between the occupation number trajectories: each time $n_{i}(\tau)$ is raised (resp. decreased) by 1 , the occupation number $n_{j}(\tau)$ of one of the neighbors $j \in \partial i$ must decrease (resp. increase) of 1 , meaning one particle has jumped from $j$ to $i$ (resp. from $i$ to $j$ ). Moreover each hopping event multiplies the weight of the trajectory $\left\{n_{i}(\tau)\right\}$ by $J$ and by a coefficient depending on the instantaneous occupation numbers of the sites involved in the 
hopping. An explicit derivation of this representation shall be given in Sec. V. Here we wish to present the results of the computation in a lighter way for the ease of the reader not interested in the technical details (we follow the methodology developed for quantum spin models in [34]).

The recursion equations of the Bose-Hubbard model defined on a tree (or on a locally tree-like graph with the assumptions of the cavity method) can be expressed in terms of the probability distribution $\eta_{\text {cav }}(\boldsymbol{y})$ of the hopping trajectories $\boldsymbol{y}$ defined on the edges of the graph. This quantity $\boldsymbol{y}$ encodes the imaginary times at which a particle has crossed the edge, and the directions of the jumps; examples are given in Fig. 5. In the case of a regular Bethe lattice of connectivity $c$ one obtains the following self-consistent equation on $\eta_{\text {cav }}(\boldsymbol{y})$,

$$
\eta_{\text {cav }}(\boldsymbol{y})=\frac{1}{z_{\text {cav }}} w_{\text {link }}(\boldsymbol{y}) \sum_{\boldsymbol{y}_{1}, \ldots, \boldsymbol{y}_{c-1}} \eta_{\text {cav }}\left(\boldsymbol{y}_{1}\right) \ldots \eta_{\text {cav }}\left(\boldsymbol{y}_{c-1}\right) w_{\text {iter }}\left(\boldsymbol{y}, \boldsymbol{y}_{1}, \ldots, \boldsymbol{y}_{c-1}\right) .
$$

For simplicity we denote here with a sum symbol what is actually an integral over continuous degrees of freedom $\boldsymbol{y}$. The explicit expressions for the weights $w$ shall be given in Sec. V, see Eqs. (46), (47). Let us emphasize the formal similarity with the Ising equivalent Eq. (14), and of course the greater complexity of the basic degree of freedom in the Bose-Hubbard case, the hopping trajectory $\boldsymbol{y}$ assuming values in a much larger space than the Ising variable $\sigma \in\{+1,-1\}$. We already mentioned this problem while discussing the related equation (21) in the coherent state basis. Compared to this latter case we are however facing now an easier problem: the number of hopping events on a given edge is a (random) number which remains finite as long as the temperature is positive (it is actually of order $\beta J)$. A single hopping trajectory $\boldsymbol{y}$ can thus be encoded as an integer $p$, i.e. the number of particle jumps occuring on this edge during the imaginary time interval $[0, \beta], p$ reals precising the imaginary times where these jumps occur, and $p$ binary variables giving the direction of the jumps, see Fig. 5. In contrast the coherent state trajectories were those of two continuous functions with a priori no compact representation. This remark opens the way to an efficient numerical method for the resolution of (27) [60]. We can indeed follow the population dynamics strategy [28, 29]. The idea of this method is to represent numerically the probability distribution $\eta_{\text {cav }}(\boldsymbol{y})$ as a (weighted) sample of a large number $\mathcal{N}_{\text {traj }}$ of hopping trajectories, namely

$$
\eta_{\text {cav }}(\boldsymbol{y})=\sum_{i=1}^{\mathcal{N}_{\text {traj }}} g_{i} \delta\left(\boldsymbol{y}-\boldsymbol{y}_{i}\right)
$$

where the $\mathcal{N}_{\text {traj }}$ weights of the trajectories are normalized according to

$$
\sum_{i=1}^{\mathcal{N}_{\text {traj }}} g_{i}=1
$$

Sampling an element $\boldsymbol{y}$ from the probability distribution $\eta_{\text {cav }}$ corresponds in this representation to extract an integer $i \in\left[1, \mathcal{N}_{\text {traj }}\right]$ with probability $g_{i}$, and setting $\boldsymbol{y}=\boldsymbol{y}_{i}$. This representation of $\eta_{\text {cav }}$ is an approximation, which yields better and better numerical accuracy when $\mathcal{N}_{\text {traj }}$ grows. The determination of a sample of weights $g_{i}$ and trajectories $\boldsymbol{y}_{i}$ which turns the representation (28) into a good approximation of the solution of (27) can be performed iteratively. To explain this point let us first rewrite the self-consistent equation (27) as

$$
\eta_{\mathrm{cav}}(\boldsymbol{y})=\sum_{\boldsymbol{y}_{1}, \ldots, \boldsymbol{y}_{c-1}} \eta_{\mathrm{cav}}\left(\boldsymbol{y}_{1}\right) \ldots \eta_{\mathrm{cav}}\left(\boldsymbol{y}_{c-1}\right) P\left(\boldsymbol{y} \mid \boldsymbol{y}_{1}, \ldots, \boldsymbol{y}_{c-1}\right) \frac{\mathcal{Z}\left(\boldsymbol{y}_{1}, \ldots, \boldsymbol{y}_{c-1}\right)}{z_{\mathrm{cav}}},
$$

where we have defined

$$
P\left(\boldsymbol{y} \mid \boldsymbol{y}_{1}, \ldots, \boldsymbol{y}_{c-1}\right)=\frac{w_{\text {link }}(\boldsymbol{y}) w_{\text {iter }}\left(\boldsymbol{y}, \boldsymbol{y}_{1}, \ldots, \boldsymbol{y}_{c-1}\right)}{\mathcal{Z}\left(\boldsymbol{y}_{1}, \ldots, \boldsymbol{y}_{c-1}\right)}, \quad \mathcal{Z}\left(\boldsymbol{y}_{1}, \ldots, \boldsymbol{y}_{c-1}\right)=\sum_{\boldsymbol{y}} w_{\text {link }}(\boldsymbol{y}) w_{\text {iter }}\left(\boldsymbol{y}, \boldsymbol{y}_{1}, \ldots, \boldsymbol{y}_{c-1}\right) .
$$

Constructed in this way $P\left(\boldsymbol{y} \mid \boldsymbol{y}_{1}, \ldots, \boldsymbol{y}_{c-1}\right)$ is a conditional probability distribution over $\boldsymbol{y}$. Given the values of the hopping trajectories $\boldsymbol{y}_{1}, \ldots, \boldsymbol{y}_{c-1}$ it is actually possible to perform an exact sampling from $P\left(\boldsymbol{y} \mid \boldsymbol{y}_{1}, \ldots, \boldsymbol{y}_{c-1}\right)$ and to compute the normalization constant $\mathcal{Z}\left(\boldsymbol{y}_{1}, \ldots, \boldsymbol{y}_{c-1}\right)$. In fact this reduces to a relatively simple single site problem: one has to construct the occupation trajectory $n(\tau)$ of a site, and the associated hopping trajectory $\boldsymbol{y}$ towards one of its neighbors, given the hopping trajectories $\boldsymbol{y}_{1}, \ldots, \boldsymbol{y}_{c-1}$ on the other adjacent edges (see Fig. 5 and recall the pictorial representation given for the Ising case in Fig. 3). The $c-1$ hopping trajectories on the upper edges impose that $n(\tau)$ changes by \pm 1 at the times particles arrive or depart from the considered central site. Between these times we shall show in Sec. V that the single site problem is described by an effective Hamiltonian $V(n)-J\left(a+a^{\dagger}\right)$; each 


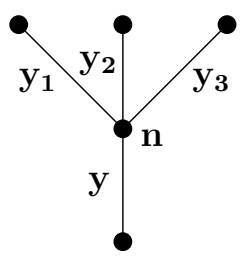

$$
\begin{aligned}
& \mathbf{y}_{\mathbf{1}}=\left(\left\lfloor\tau_{\mathbf{1}}^{\text {in }},\right\rfloor \tau_{\mathbf{2}}^{\text {in }},\left\lceil\tau_{\mathbf{4}}^{\text {in }}\right)\right. \\
& \mathbf{y}_{\mathbf{2}}=(\emptyset) \\
& \mathbf{y}_{\mathbf{3}}=\left(\left\lfloor\tau_{\mathbf{3}}^{\text {in }}\right)\right. \\
& \mathbf{y}=\left(\left\lfloor\tau_{\mathbf{1}}^{\text {out }},\left\lfloor\tau_{\mathbf{2}}^{\text {out }}\right)\right.\right.
\end{aligned}
$$

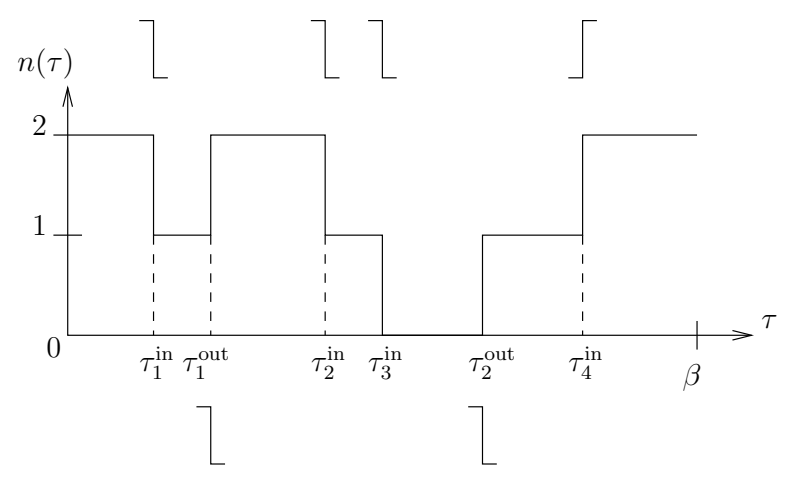

FIG. 5: Example of the sampling process from the law $P\left(\boldsymbol{y} \mid \boldsymbol{y}_{1}, \ldots, \boldsymbol{y}_{c-1}\right)$ defined in Eq. (31) for $c=4$. The $c-1$ incoming hopping trajectories $\left(\boldsymbol{y}_{1}, \boldsymbol{y}_{2}, \boldsymbol{y}_{3}\right)$ impose jumps in $\tau_{1}^{\text {in }}<\tau_{2}^{\text {in }}<\tau_{3}^{\text {in }}<\tau_{4}^{\text {in }}$, a particle arriving on the central site at the first three imaginary times, leaving at the fourth one, corresponding to the symbols in the upper part of the rightmost figure. A trajectory $n(\tau)$ respecting these constraints is shown; it contains two other jumps at $\tau_{1}^{\text {out }}<\tau_{2}^{\text {out }}$, associated to hopping events in $\boldsymbol{y}$. The symbols on the bottom are reversed: particles leaving the central site will arrive on the next level of the recursive equation.

change in the value of $n(\tau)$ provoked by the creation/annihilation operators in this effective Hamiltonian is associated to an hopping event in the new (downwards) trajectory $\boldsymbol{y}$. An example of this construction is given in Fig. 5. The computation of $\mathcal{Z}\left(\boldsymbol{y}_{1}, \ldots, \boldsymbol{y}_{c-1}\right)$ can be performed recognizing it as the partition function of the single-site effective Hamiltonian.

Suppose now a representation of $\eta_{\text {cav }}$ is available under the form (28); one can plug it into the right-hand-side of Eq. (30) and construct a new set of trajectories and weights corresponding to its left-hand-side, let us call them $\left\{\boldsymbol{y}_{j}^{\prime}, g_{j}^{\prime}\right\}_{j=1}^{\mathcal{N}_{\text {traj }}}$. Independently and identically for each $j \in\left[1, \mathcal{N}_{\text {traj }}\right]$, this can be done by:

- drawing $c-1$ integers $i_{1}, \ldots, i_{c-1} \in\left[1, \mathcal{N}_{\text {traj }}\right]$, each of them independently with probability $g_{i}$

- drawing $\boldsymbol{y}$ from $P\left(\boldsymbol{y} \mid \boldsymbol{y}_{i_{1}}, \ldots, \boldsymbol{y}_{i_{c-1}}\right)$

- setting $\boldsymbol{y}_{j}^{\prime}=\boldsymbol{y}, g_{j}^{\prime}=\mathcal{Z}\left(\boldsymbol{y}_{i_{1}}, \ldots, \boldsymbol{y}_{i_{c-1}}\right)$

The new weights $g_{j}^{\prime}$ are then normalized to fulfill Eq. (29). This process can be iterated, plugging in the r.h.s. of Eq. (30) the newly obtained representation $\left\{\boldsymbol{y}_{j}^{\prime}, g_{j}^{\prime}\right\}$, and so on and so forth. After a certain number of steps, starting from an arbitrary initial condition[61], a stationary solution is reached.

The physical observables can be computed from this representation of $\eta_{\text {cav }}$. Let us first explain the determination of the average occupation number of one site. Following our convention we denote $\boldsymbol{n}$ the occupation number imaginarytime trajectory $n(\tau)$. Its probability distribution $\eta(\boldsymbol{n})$ is expressed by considering the complete environment of a vertex with its $c$ neighbors, as was done for the local magnetization of the Ising model in Eq. (15) and schematized in Fig. 4,

$$
\eta(\boldsymbol{n})=\frac{1}{z_{\text {site }}} \sum_{\boldsymbol{y}_{1}, \ldots, \boldsymbol{y}_{c}} \eta_{\text {cav }}\left(\boldsymbol{y}_{1}\right) \ldots \eta_{\text {cav }}\left(\boldsymbol{y}_{c}\right) w_{\text {site }}\left(\boldsymbol{n}, \boldsymbol{y}_{1}, \ldots, \boldsymbol{y}_{c}\right)
$$

The explicit expression of the weight $w_{\text {site }}$ shall be given in Sec. V. It is however intuitive that it will contain a factor $\exp \left[-\int \mathrm{d} \tau V(n(\tau))\right]$ arising from the local part of the Hamiltonian, and requires a consistency between $\boldsymbol{n}$ and $\left(\boldsymbol{y}_{1}, \ldots, \boldsymbol{y}_{c}\right)$. In fact all the discontinuities in the occupation trajectory $n(\tau)$ are fixed by the hopping events on the $c$ neighboring edges. If the number of hoppings towards the considered site equals the number of jumps outside, then the trajectory $n(\tau)$ is fixed up to a global shift $n(\tau) \rightarrow n(\tau)+m$ with $m$ independent of time. Whenever there is an unbalanced number of hopping towards/outside the vertex no periodic trajectory $n(\tau)$ can be constructed, $w_{\text {site }}=0$ for such a configuration of $\left(\boldsymbol{y}_{1}, \ldots, \boldsymbol{y}_{c}\right)$. The average occupation number of a site is easily obtained from this probability $\eta(\boldsymbol{n})$,

$$
\left\langle a^{\dagger} a\right\rangle=\sum_{\boldsymbol{n}} \eta(\boldsymbol{n}) n(0)=\frac{\sum_{\boldsymbol{y}_{1}, \ldots, \boldsymbol{y}_{c}} \eta_{\mathrm{cav}}\left(\boldsymbol{y}_{1}\right) \ldots \eta_{\mathrm{cav}}\left(\boldsymbol{y}_{c}\right) \sum_{\boldsymbol{n}} w_{\mathrm{site}}\left(\boldsymbol{n}, \boldsymbol{y}_{1}, \ldots, \boldsymbol{y}_{c}\right) n(0)}{\sum_{\boldsymbol{y}_{1}, \ldots, \boldsymbol{y}_{c}} \eta_{\mathrm{cav}}\left(\boldsymbol{y}_{1}\right) \ldots \eta_{\mathrm{cav}}\left(\boldsymbol{y}_{c}\right) \sum_{\boldsymbol{n}} w_{\text {site }}\left(\boldsymbol{n}, \boldsymbol{y}_{1}, \ldots, \boldsymbol{y}_{c}\right)},
$$




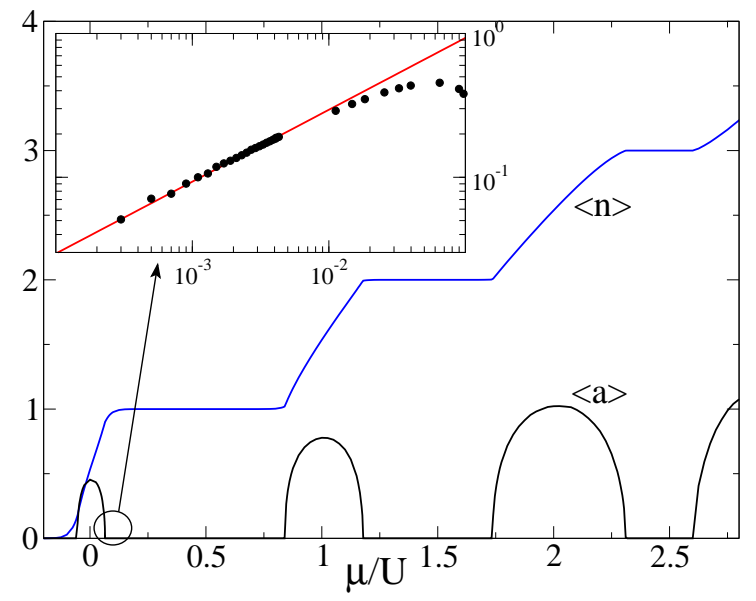

FIG. 6: (Main panel) $\langle n\rangle$ and $\langle a\rangle$ as functions of $\mu / U$ for $J / U=0.02$, at $\beta J=1$ and $N_{\text {cut }}=6$ for Bethe lattices of connectivity $c=4$. Full lines are obtained by joining points measured every $\Delta \mu / U \sim 0.05$, except close to the critical points where $\Delta \mu / U \sim 0.001$. (Inset) Blow-up of the region close to the first SF-MI transition that happens at $\mu_{c}=0.0648 U$. Here $\langle a\rangle$ is plotted as a function of $\delta=\left(\mu_{c}-\mu\right) / U$ (circles). The full line is a fit to $\langle a\rangle \sim A \delta^{0.5}$ with $A=2.9475$.

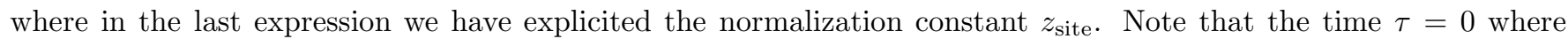
$n(\tau)$ is evaluated is arbitrary because of the cyclic invariance along the imaginary time axis, hence $n(0)$ can be equivalently replaced by the temporal average $\frac{1}{\beta} \int_{0}^{\beta} \mathrm{d} \tau n(\tau)$. Given a representation of $\eta_{\text {cav }}$ as a weighted sample (28) the numerical determination of $\left\langle a^{\dagger} a\right\rangle$ is straightforward. Both the numerator and the denominator of (33) have the form of the average of a function $u\left(\boldsymbol{y}_{1}, \ldots, \boldsymbol{y}_{c}\right)$ with the $\boldsymbol{y}$ 's independently drawn from their distribution $\eta_{\text {cav }}$. As already explained drawing from $\eta_{\text {cav }}$ a trajectory $\boldsymbol{y}$ amounts to draw an index $i \in\left[1, \mathcal{N}_{\text {traj }}\right]$ with probability $g_{i}$ and picking the element $\boldsymbol{y}_{i}$ of the population. In formula this method of sampling leads to

$$
\sum_{\boldsymbol{y}_{1}, \ldots, \boldsymbol{y}_{c}} \eta_{\text {cav }}\left(\boldsymbol{y}_{1}\right) \ldots \eta_{\text {cav }}\left(\boldsymbol{y}_{c}\right) u\left(\boldsymbol{y}_{1}, \ldots, \boldsymbol{y}_{c}\right)=\frac{1}{\mathcal{N}_{\text {tries }}} \sum_{j=1}^{\mathcal{N}_{\text {tries }}} u\left(\boldsymbol{y}_{i_{j, 1}}, \ldots, \boldsymbol{y}_{i_{j, c}}\right),
$$

where $\left\{i_{j, l}\right\}$ are integers drawn independently with the probability $g_{i}$, with $j \in\left[1, \mathcal{N}_{\text {tries }}\right]$ and $l \in[1, c]$. The numerical accuracy of the sampling is increased by taking a large value of $\mathcal{N}_{\text {tries }}$. Moreover one can interleave average steps with iteration steps, determining a new set of weights $g_{i}$ and trajectories $\boldsymbol{y}_{i}$ and recomputing independent averages on this new representation of $\eta_{\text {cav }}$.

We shall show in Sec. V that all the other observables defined in Sec. II (order parameter $\langle a\rangle$, free-energy, kinetic/potential energy and Green function) can also be expressed as averages of the form (34), and thus can be efficiently computed by the method developed here.

\section{B. Results}

In the following we present the results obtained for the Bose-Hubbard model on the Bethe lattice solved with the cavity method. We have shown in the previous sections that in the thermodynamic limit the exact resolution of the model amounts to solve Eq. (27) and we explained how this can be done with arbitrary numerical precision using the population dynamics algorithm described in subsection IV A, encoding $\eta_{\text {cav }}(\boldsymbol{y})$ as a population of a large number $\mathcal{N}_{\text {traj }}$ of trajectories. We typically used $\mathcal{N}_{\text {traj }}=32768$ and checked that the results were unchanged when using larger $\mathcal{N}_{\text {traj. }}$. We recall that in the thermodynamic limit the local observables (say, $\left\langle n_{i}\right\rangle$ ) are independent on the site $i$. In the following we focus on Bethe lattices of connectivity $c=4$ and 6 , which mimic the connectivity of the two and three-dimensional square and cubic lattices, respectively.

Note that in order to implement the summations over $n$ involved in the computation of the various weights appearing in Eqs. (27) and (32), we have to introduce a cutoff on the possible values of $n, 0 \leq n<N_{\text {cut }}$. The technical details will be discussed in section V. Since all the results presented below corresponds to regions of the parameter space such that $0 \leq\langle n\rangle \leq 3$, we work with a cut-off on the occupation number in the interval $4 \leq N_{\text {cut }} \leq 6$. We have checked for various values of the parameters that all the results are stable with respect to changes of the cutoff (in some cases also up to $N_{\text {cut }}>6$ ). 


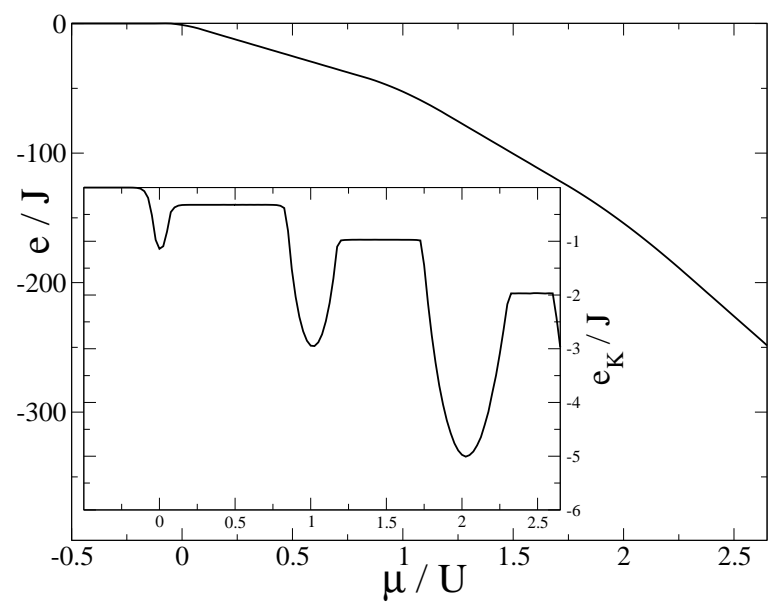

FIG. 7: (Main panel) Total energy, $e=\langle H\rangle / N$, as a function of $\mu / U$ for $J / U=0.02$, at $\beta J=1$ and $N_{\text {cut }}=6$ for Bethe lattices of connectivity $c=4$. (Inset) Kinetic energy $e_{K}$ as a function of the chemical potential for the same values of the parameters.

In this section we also compare our results with other analytic approaches, such as the variational mean-field treatment described in Sec. II B and the bosonic Dynamical Mean Field Theory (B-DMFT)[62] discussed in Sec. III C, that correspond to the large $c$ limit of our method [23,24]. We also compare them to Quantum Monte Carlo simulations on $2 d$ square lattice [17] and $3 d$ cubic lattice [18].

\section{Thermodynamic observables}

Once the numerical iterative procedure has converged to a fixed point solution, one can compute the thermodynamic observables. Fig. 6 shows the behavior of $\langle n\rangle$ and $\langle a\rangle$, as a function of the chemical potential, $\mu / U$ for $J / U=0.02$, and $\beta J=1$ for a Bethe lattice of connectivity $c=4$. We have also computed $\langle n\rangle$ and $\langle a\rangle$ at lower values of the temperature $(\beta J=2,4$, and 6$)$, but we find that the results are unchanged within our numerical accuracy, except very close to the lowest- $\mu$ superfluid-insulator transitions, where a little temperature effect is detected[63] between $\beta J=1$ and $\beta J=2$ (while no change is detectable above $\beta J=2$ ). Hence, we can safely assume that for $\beta J \geq 2$ we are in the zero temperature regime.

The plot of Fig. 6 clearly shows a sequence of transitions from superfluid phases (SF) to Mott insulators (MI). The superfluid phase is characterized by a finite value of $\langle a\rangle$, which corresponds to a finite condensate fraction $f_{\mathrm{BEC}}=\langle a\rangle^{2} /\langle n\rangle$, while in the Mott insulator $\langle a\rangle=0$. In the limit of zero temperature in the MI regions the average number of bosons per site, $\langle n\rangle$ is fixed to integer values, $\langle n\rangle=1,2,3, \ldots$. As a result, the compressibility of the system, $\chi=\partial\langle n\rangle / \partial \mu$ vanishes identically. At small but finite temperature, we expect the compressibility to be exponentially small in the temperature, and indeed in Fig. 6 we observe that the compressibility at commensurate density is practically zero even at $\beta J=1$.

As shown in the inset of Fig. 6, the critical exponent for $\langle a\rangle \sim\left(\mu_{c}-\mu\right)^{\epsilon}$ at the transition from the SF phase to the MI is consistent with the mean-field value $\epsilon=1 / 2$. As in the classical case, the critical exponents found on the Bethe lattice are equal to the mean-field ones (see [34] for a detailed discussion in the case of the quantum spin-1/2 ferromagnet on the Bethe lattice). Therefore, we also expect the other critical exponents to coincide with their mean-field values.

In Fig. 7 we plot the total energy, $e=\langle H\rangle / N$, as a function of the chemical potential for the same values of the parameters as in Fig. 6. We have also computed the free energy of the system, $f$, but we do not show it since at these low temperatures the difference between the energy and the free energy is very small, and practically undetectable within our numerical precision, which confirms that we are effectively in the zero-temperature regime and the system can be considered to be in its ground state. In the inset of Fig. 7 we show the behavior of the kinetic energy, $e_{K}$ as a function of $\mu / U$. The kinetic energy is, of course, lower in the SF phase than in the MI. Moreover we find that $e_{K}$ does not vanish in the MI phase, differently from the traditional mean-field approach, where hopping is completely suppressed in this phase. Note that both the kinetic energy and the potential energy show a discontinuity in the first derivative at the transition between the MI and the SF phase. On the contrary, the first derivative of the total energy (and of the free energy as well) is continuous at the transition; only its second derivative shows a discontinuity. 

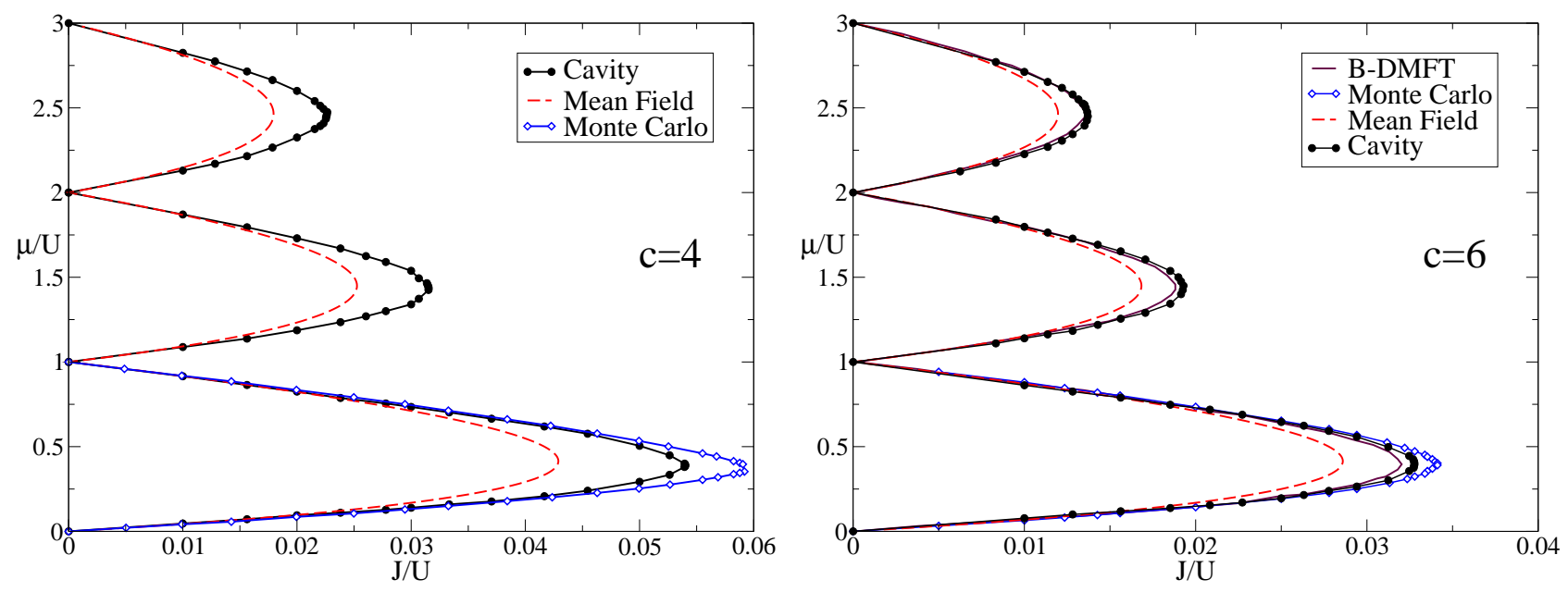

FIG. 8: "Zero temperature" phase diagram of the Bose-Hubbard model on the Bethe lattice in the $(J / U, \mu / U)$ plane at connectivity $c=4$ (left panel) and $c=6$ (right panel). The results obtained within the cavity method (black lines and filled circles) are compared to Monte Carlo simulation (blue lines and open diamonds) on the square (data from [17]) and the cubic (data from [18]) lattice, and to the prediction of the mean-field approach (red dashed curves) and of bosonic DMFT (brown full curve in right panel, data from [24]).

\section{Phase diagram}

In Fig. 8 we present the "zero temperature" (recall that our method only works at finite temperature, hence we use quotes to remind that an extrapolation to $T=0$ is needed) phase diagram of the Bose-Hubbard model on the Bethe lattice of connectivity $c=4$ (left panel) and $c=6$ (right panel) in the $(J / U, \mu / U)$ plane. The phase boundaries have been obtained by computing $\langle a\rangle$ as a function of $\mu / U$ at constant $J / U$. In this case we used $4 \leq N_{\text {cut }} \leq 6$ and $4 \leq \beta J \leq 6$ (lower values of $\beta J$ and higher values of $N_{\text {cut }}$ have been used at higher $\mu$ ). As already discussed, at these values of $\beta$ we are effectively in the zero temperature regime. The lobelike shape of the phase boundary between MI and SF phases is qualitatively similar to the one found in the mean field approach. In Fig. 8 we also compare the results found with the quantum cavity method with the outcomes of Quantum Monte Carlo simulations (on the square lattice for $c=4$ [17] and on the cubic lattice for $c=6[18]$ ), and with the results of other analytical approaches. This comparison shows that the cavity method does a fairly good job in locating the lobelike contours between the MI and the SF phase and performs much better than the mean-field approach. It also performs slightly better than the Bosonic DMFT [24] for $c=6$, although the difference between the cavity method and the B-DMFT is expected to become small as the connectivity is increased. It would be interesting in this respect to compare the cavity method and B-DMFT for $c=4$.

In addition to the low temperature phase diagram we have computed the transition temperature line from the "insulating" phase (which we defined by $\langle a\rangle=0$, but has still a finite conductivity at finite temperature) to the superfluid one (defined by $\langle a\rangle \neq 0$ ) at unit filling $\left(\left\langle a^{\dagger} a\right\rangle=1\right.$ ), for Bethe lattices of connectivity $c=6$. This result is plotted in Fig. 9 and compared to the mean-field prediction and to the three-dimensional Monte-Carlo simulations of $[18]$.

\section{Green functions and particle occupation statistics}

As far as the results shown up to now are concerned, there are no qualitative differences between the Bethe lattice and the fully connected models (or equivalently the mean field approximation). We shall now present the results for the one-particle on-site imaginary time Green function, which exhibits a richer behavior with respect to the mean-field description. This quantity, defined in Eq. $(6)$ as $G(\tau)=\left\langle T a(\tau) a^{\dagger}(0)\right\rangle$, is plotted in Fig. 10 for two set of values of the parameters $(J / U, \mu / U)$, one in the MI phase, the other in the SF, both very close to the tip of the first insulating lobe.

Let us first remark that the decay of the Green function is independent on temperature in this temperature range, the effect of temperature being only to cut the decay around $|\tau| \sim \beta / 2$ due to periodicity. Therefore we can safely assume that the Green functions reported in Fig. 10 are good estimates of their zero-temperature limit. 


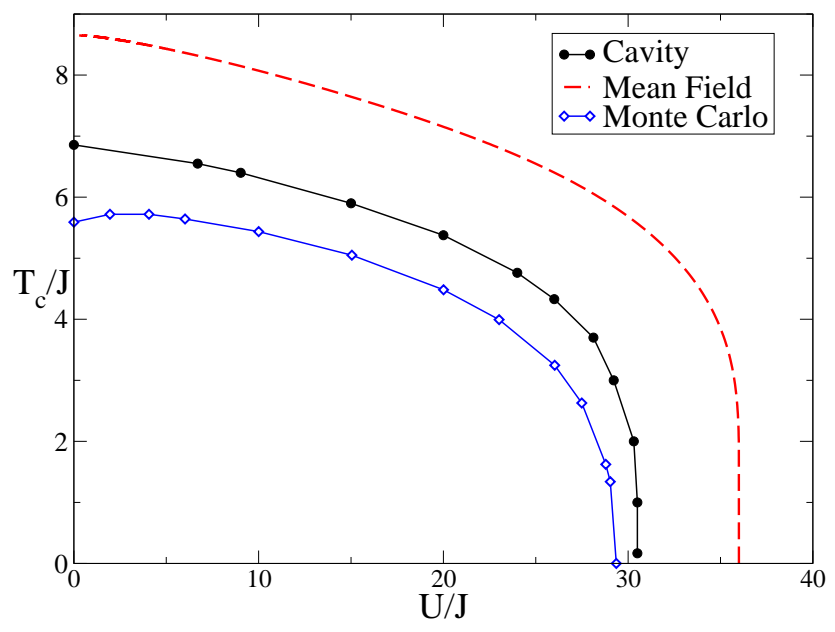

FIG. 9: Interaction dependence of the transition temperature at unit filling. The result obtained on the $c=6$ Bethe lattice (black lines and filled circles) is compared to Monte Carlo simulation (blue lines and open diamonds) on the cubic lattice (data from [18]), and to the prediction of the mean-field approximation (red dashed curves).

For the interpretation of these curves it is worth recalling the spectral representation of the Green function [35] at zero temperature: for $\tau>0, G(\tau)=|\langle a\rangle|^{2}+\int_{0}^{\infty} \rho_{p}(E) \exp (-E \tau)$, where $\rho_{p}(E)$ is the (on-site) spectral density for particle excitations. A similar expression holds for $\tau<0$ in terms of hole excitations. The long time limit of $G(\tau)$ is thus strictly positive in the SF phase, and we checked that is in agreement with the value $|\langle a\rangle|^{2}$ previously computed (see the dotted line in the right panel of Fig. 10).

Let us now turn to the discussion of the Green function in the MI. In the mean-field description of this phase the hopping is completely suppressed, hence particle (resp. hole) excitations corresponds to the addition (resp. removal) of one particle from the background of the commensurate filling of immobile particles. To be concrete let us consider the first lobe with $\langle n\rangle=1$; the energy of these particle and hole excitations are easily found to be $E_{p}=U-\mu$ and $E_{h}=\mu$, hence follows the expression of the Green function [45]:

$$
G_{\mathrm{mf}}(\tau)=\theta(\tau) 2 e^{-(U-\mu) \tau}+\theta(-\tau) e^{-\mu \tau} .
$$

In other words the spectral density of particle excitations is made of a single delta function (and similarly for the hole excitations). The mean-field Green function is reported in Fig. 10 as a dashed black line; the comparison with the results of the Bethe lattice computations shows that the latter is more complex. The decay of the latter is not a simple exponential, thus reflecting a non-trivial spectrum of excitations. Moreover the energy of particle and hole excitations is lower on the Bethe lattice, as can be seen from their slower decay. This happens because on the Bethe lattice, due to the local finite connectivity, particles can still hop around even in the MI phase, resulting in a gain of kinetic energy and a lowering of the energy cost of the excitations. Note that different improvements of the mean-field treatment, for instance Random Phase Approximations (RPA) [13, 14], provide a much better description of the spectral density in the Mott phase, in particular a good approximation to the wave-vector dependence of the excitation energies for finite-dimensional lattices. A quantitative comparison with the predictions of the RPA is beyond the scope of this paper. We focused instead on the comparison with the fully-connected result and B-DMFT, because they are related to the Bethe lattice via a well-controlled limit, namely the large connectivity one.

We did not attempt to perform the inverse Laplace transform to determine the spectral function, yet we performed the following analysis to estimate the scale $E_{p}$ of the slowest decay in the $\tau>0$ part of the Green function. A plot of $\log \left[\tau^{\alpha} G(\tau)\right] / \tau$ is seen to approach a constant at large $\tau$ for $\alpha \sim 1$, suggesting that $G(\tau)$ decays as $\exp \left(-E_{p} \tau\right) / \tau$, hence that the spectral function $\rho_{p}(E)$ is finite at $E=E_{p}$ and vanishes for $E<E_{p}$. This is consistent with the results of $[13,14]$. For simplicity, we have chosen to fit the Green function to $G(\tau)=2 e^{-E_{p} \tau}\left(1-e^{-\Delta_{p} \tau}\right) /\left(\Delta_{p} \tau\right)$, corresponding to a flat density of states in $\left[E_{p}, E_{p}+\Delta_{p}\right]$. We stress that this is an arbitrary choice that we made only to fit the long time behavior of $G(\tau)$ and determine $E_{p}$. Note that at very small $\tau \ll 1 / J$, hopping is irrelevant and $G(\tau) \sim G_{\mathrm{mf}}(\tau)$ at first order in $\tau$. This imposes a relation between $\Delta_{p}$ and $E_{p}$, hence there is a single free parameter in our fitting procedure.

In Fig. 11 we show the behavior of the Green function on approaching the phase transition from the MI phase, close to the tip of the first lobe by varying $J / U$ at constant $\mu / U=0.39$. We focus on positive $\tau$ and fix $\beta J=6$, which according to Fig. 10 is a low enough temperature, such that the Green function is identical to its zero- 

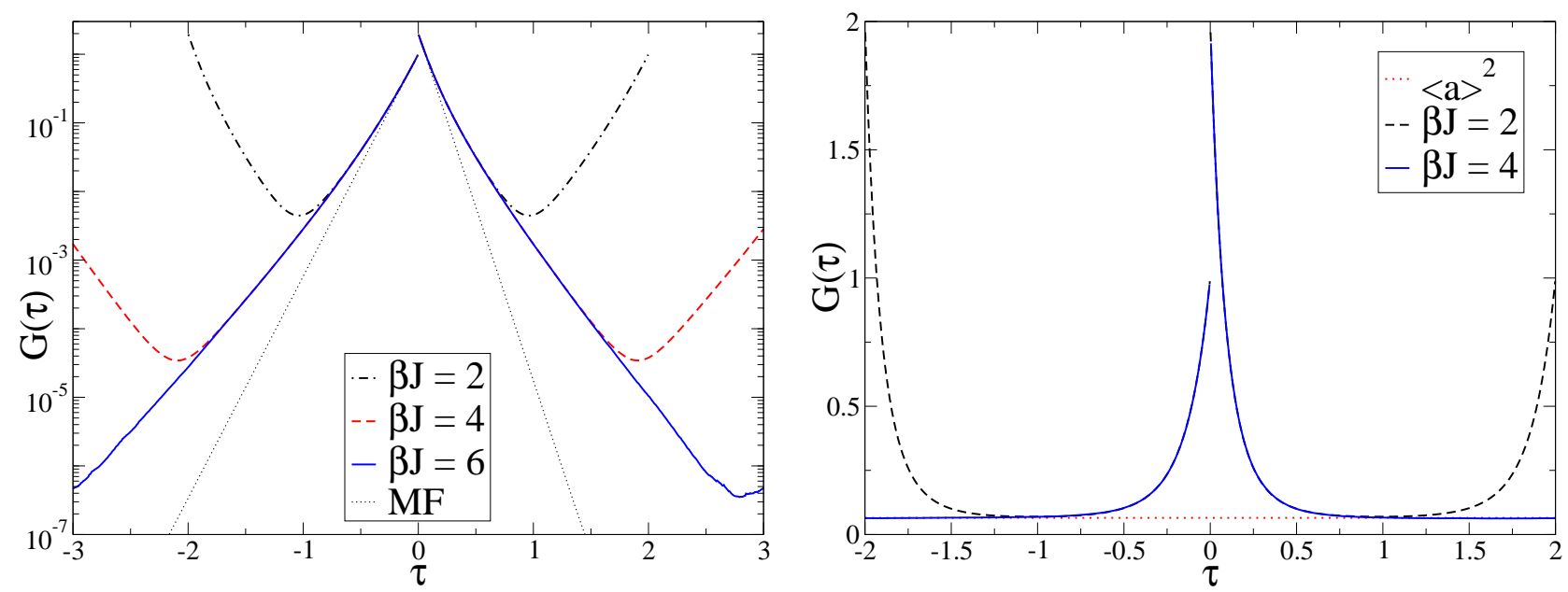

FIG. 10: Green function $G(\tau)$ as a function of the imaginary time at $c=4$. (Left panel) Results in the MI phase, at $J / U=0.0523, \mu / U=0.39, N_{\text {cut }}=4$ and $\beta J=2$ (black dashed-dotted curve), 4 (red dashed curve), and 6 (blue continuous curve). Also reported is the mean-field result for the same parameters (black dotted curve). (Right panel) Results in the SF phase, at $J / U=0.0555, \mu / U=0.39, N_{\text {cut }}=6$ and $\beta J=2$ (black dashed curve) and 4 (blue curve). Also reported is the long time limit of $G(\tau)$, which corresponds to the value of $\langle a\rangle^{2}$ for these values of the parameters.

\begin{tabular}{|c|c|c|c|c|c|c|}
\hline$n$ & 0 & 1 & 2 & 3 & 4 & 5 \\
\hline$P_{n}(\mathrm{SF})$ & 0.034 & 0.93 & 0.034 & 0.00027 & $5.7 \mathrm{e}-07$ & $2.2 \mathrm{e}-11$ \\
$P_{n}(\mathrm{MI})$ & 0.023 & 0.95 & 0.023 & 0.00013 & & \\
\hline
\end{tabular}

TABLE I: Values of $P_{n}$ in the MI and in the SF, for the same values of the parameters as in Fig. 10, namely $J / U=0.0523$, $\mu / U=0.39, N_{\text {cut }}=4$ and $\beta J=4$ (in the MI phase) and $J / U=0.0555, \mu / U=0.39, N_{\text {cut }}=6$ and $\beta J=4$ (in the SF phase).

temperature limit for $\tau<\beta / 2$. We use the rescaled time variable $\tau U$, in such a way that the mean field result $G_{\mathrm{mf}}(\tau)=2 \exp [-\tau U(1-\mu / U)]$ yields the same decay at all values of $J / U$. On the contrary, the Bethe lattice Green function depends quite strongly on $J / U$, and its decay slows down on approaching the transition. The result of the fitting procedure explained above is shown in the inset of Fig. 11 and shows that the parameter $E_{p}$ is smaller by a factor of 2 on the Bethe lattice compared to the mean field results; it decreases on approaching the transition, but remains finite at the transition. Indeed we have computed here the on-site Green-function, which reflects only the localized single-particle excitations, whereas the transition towards the superfluid phase is towards a delocalized state (small momentum in the finite dimensional case [18]). To detect the signature of the transition in such a way it would be necessary to compute the spatial and temporal dependence of the Green function, which is in principle possible using the cavity method (see next subsection).

We expect (but did not check in detail) that also in the SF phase the on-site Green function will decay exponentially up to the transition point. This shows also that the BEC state on the Bethe lattice has a peculiar nature as compared to the finite dimensional case: condensation happens in the uniform state, which is separated from the other eigenvalues of the connectivity matrix by a gap [46, 47]. In particular, one can check explicitly by a Bogoliubov-like computation [48] that the single-particle excitation spectrum is gapped at small $U / J$.

The difference between the mean field approach and the Bethe lattice result is also unveiled by the analysis of the probability distribution $P_{n}$, already studied recently in [19] using Monte Carlo simulations, defined as the probability to detect $n$ bosons on a given lattice site:

$$
P_{n_{0}}=\left\langle\delta_{n, n_{0}}\right\rangle=\frac{1}{Z} \operatorname{Tr}\left[\delta_{n, n_{0}} e^{-\beta H}\right] .
$$

This is another quantity sensitive to the occupation number fluctuations. In table I we report the values of $P_{n}$ in the $\mathrm{MI}$ and in the SF, for the same values of the parameters as for Fig. 10. These data clearly indicate that even in the MI phase the occupation number of a given lattice site is not strictly fixed to an integer value, as in the mean field approach: there is still a finite probability of finding a number of bosons different from one within the lobe at $\langle n\rangle=1$. Interestingly enough, these quantities can be probed in experiments [49]. 


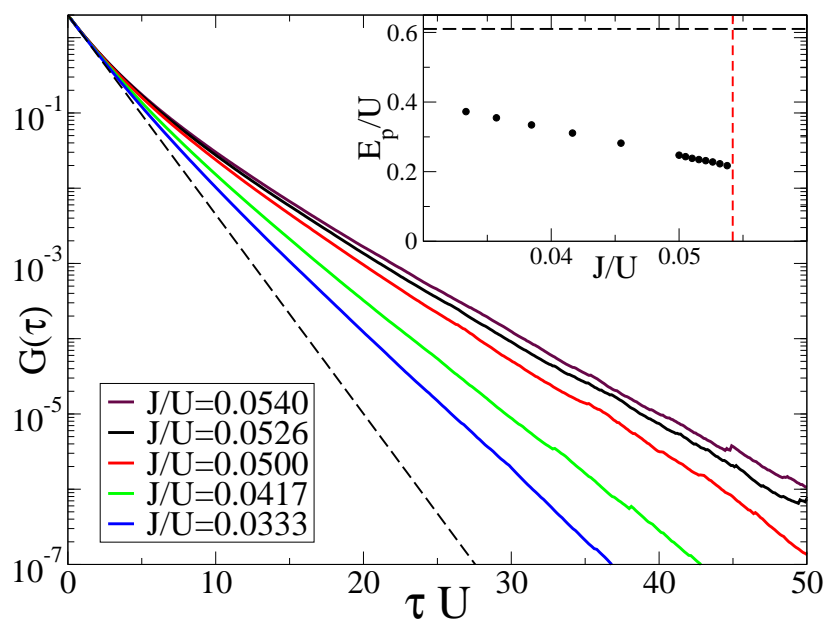

FIG. 11: (Main Panel) Green function $G(\tau)$ as a function of the rescaled imaginary time $\tau U$ for $c=4$ in the MI phase, close to the tip of the first lobe at $\mu / U=0.39, N_{\text {cut }}=4$ and $\beta J=6$, at different values of $J / U$ (approaching the transition to the superfluid, from bottom to top). Also reported is the mean-field result for the same parameters (black dashed curve). (Inset) Particle excitation gap $E_{p} / U$ is plotted as a function of $J / U$ (see text for details); the dashed black line is the mean field result $E_{p} / U=1-\mu / U$, the vertical line marks the transition to the superfluid.

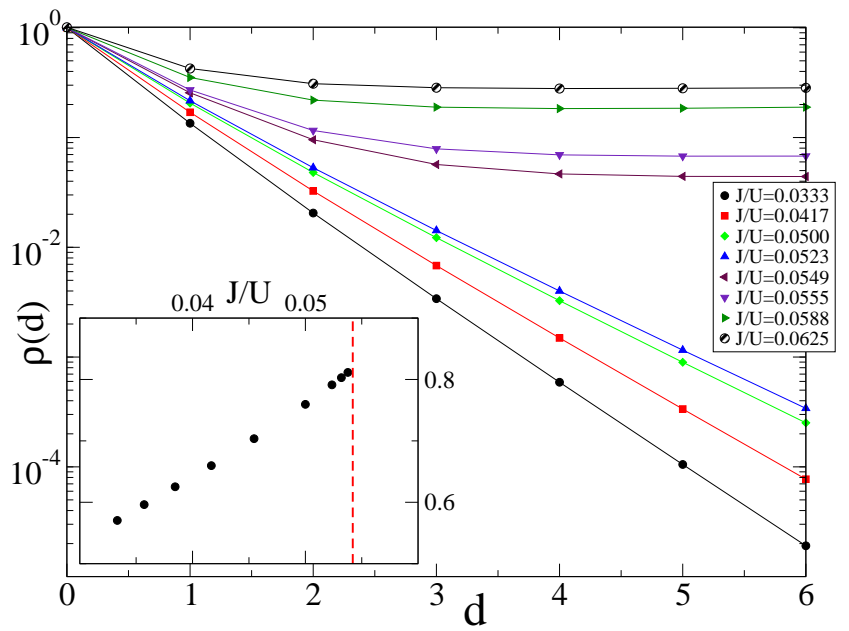

FIG. 12: (Main Panel) One particle density matrix $\rho_{d}=\left\langle a_{0}^{\dagger} a_{d}\right\rangle$ at $c=4$, fixed $\mu / U=0.39, \beta J=2, N_{\text {cut }}=4$, and different values of $J / U$ crossing the tip of the first MI lobe in Fig. 8. In the MI phase $\rho_{d}$ decays to zero, while in the SF phase it decays to $|\langle a\rangle|^{2}$. (Inset) Correlation length as a function of $J / U$ in the MI phase. The vertical line marks the MI-SF transition.

\section{One particle density matrix}

We present now our results for the one-particle density matrix $\rho_{i j}=\left\langle a_{i}^{\dagger} a_{j}\right\rangle$. Because of the invariance structure of the Bethe lattice this will depend (in the thermodynamic limit) only on the distance between the sites $i$ and $j$, that we denoted as $d(i, j)$ in the discussion of Sec. III A. In Fig. 12 we report $\rho_{d}$ at different points in the $(J / U, \mu / U)$ plane, crossing the insulator-superfluid transition around the tip of the first lobe.

In the MI phase $\rho_{d}$ decays to zero, while in the SF phase we checked that it correctly decays to $|\langle a\rangle|^{2}$. The decay is always quite fast, and in the SF phase we do not have enough points to perform a reliable fit to extract a correlation length, altought it is clear that the decay becomes slower and slower on approaching the transition to the MI. On the contrary, in the MI phase it is very easy to extract the correlation length by measuring the slope of $\log \rho_{d}$ versus $d$ for $d \geq 3$. The result is plotted in the inset of Fig. 12 and shows that the correlation length increases also on the MI side. 
It might be surprising at first sight that the correlation length stays finite at the transition; however, as we discussed at the end of section III A, this is a general property of Bethe lattices that is related to the fact that the number of sites $\mathcal{N}_{d}$ at distance $d$ from a given one scales as $(c-1)^{d}$ for large $d$. Despite this difference at criticality, we believe that the possibility of investigating spatial correlations on the Bethe lattice is very interesting away from critical points, and is an important improvement of the cavity method with respect to the mean field approximation.

\section{DETAILED DERIVATION}

\section{A. Lattice boson models in the Suzuki-Trotter formalism}

This section is devoted to a complete derivation of the equations used in Sec. IV. We shall consider a slightly more general Hamiltonian than (1),

$$
H=-\sum_{\langle i, j\rangle} J_{\langle i, j\rangle}\left(a_{i}^{\dagger} a_{j}+a_{j}^{\dagger} a_{i}\right)+\sum_{i=1}^{N} V_{i}\left(a_{i}^{\dagger} a_{i}\right)
$$

where the hopping strength $J_{\langle i, j\rangle}$ is allowed to vary from edge to edge and we consider arbitrary local potential energies $V_{i}(n)$. This will be convenient both for notational reasons and because part of the following discussion will also apply to the disordered Bose-Hubbard model. The original model (1) is recovered by taking $J_{\langle i, j\rangle}=J$ on all links, and $V_{i}(n)=\frac{U}{2} n(n-1)-\mu n$. The Hilbert space of the model is spanned by the occupation number vectors $\left|n_{1}, \ldots, n_{N}\right\rangle$, where $n_{i}$ is a positive or null integer counting the number of particles on site $i$. We shall use the more compact notation $|\underline{n}\rangle=\left|n_{1}, \ldots, n_{N}\right\rangle$ to denote one of these basis vectors. For completeness we recall the action of the annihilation $a_{i}$ and creation $a_{i}^{\dagger}$ operators in this basis,

$$
\begin{aligned}
a_{i}|\underline{n}\rangle & =\sqrt{n_{i}}\left|n_{1}, \ldots, n_{i-1}, n_{i}-1, n_{i+1}, \ldots, n_{N}\right\rangle, \\
a_{i}^{\dagger}|\underline{n}\rangle & =\sqrt{n_{i}+1}\left|n_{1}, \ldots, n_{i-1}, n_{i}+1, n_{i+1}, \ldots, n_{N}\right\rangle .
\end{aligned}
$$

The number operators $a_{i}^{\dagger} a_{i}$ are diagonal in this basis, with $a_{i}^{\dagger} a_{i}|\underline{n}\rangle=n_{i}|\underline{n}\rangle$. The partition function of the Hamiltonian (37) will be computed using the Suzuki-Trotter formula (18), cutting the imaginary time axis of length $\beta$ in a number $N_{\mathrm{s}}$ (ultimately sent to infinity) of slices. At each of these slices a representation of the identity in the occupation number basis is inserted. This yields

$$
Z=\operatorname{Tr}\left[e^{-\beta H}\right]=\lim _{N_{\mathrm{s}} \rightarrow \infty} \sum_{\underline{n}^{1}, \cdots, \underline{n}^{N_{\mathrm{s}}}} \exp \left[-\frac{\beta}{N_{\mathrm{s}}} \sum_{\alpha=1}^{N_{\mathrm{s}}} \sum_{i=1}^{N} V_{i}\left(n_{i}^{\alpha}\right)\right] \prod_{\alpha=1}^{N_{\mathrm{s}}}\left\langle\underline{n}^{\alpha}\left|e^{\frac{\beta}{N_{\mathrm{s}}} \sum_{\langle i, j\rangle} J_{\langle i, j\rangle}\left(a_{i}^{\dagger} a_{j}+a_{j}^{\dagger} a_{i}\right)}\right| \underline{n}^{\alpha+1}\right\rangle .
$$

The index $\alpha$ is the discrete coordinate between 1 and $N_{\mathrm{s}}$ along the imaginary time axis, and we use periodic boundary conditions $\underline{n}^{N_{s}+1}=\underline{n}^{1}$. The quantum problem has thus been transformed in a classical one, in terms of the imaginarytime dependent classical variables $\underline{n}^{\alpha}$. The expression above of the hopping interaction is however unpractical for our future needs, we shall thus transform it by introducing a set of auxiliary variables $\underline{y}^{\alpha}=\left\{y_{i \rightarrow j}^{\alpha}\right\}$ which can take values 0 or 1 , depend on the discrete time $\alpha$, and are defined on the directed edges of the graph (an edge $\langle i, j\rangle$ thus bears, for each time $\alpha$, two variables $y_{i \rightarrow j}^{\alpha}$ and $y_{j \rightarrow i}^{\alpha}$ ). It is simple to show that

$$
\begin{aligned}
\left\langle\underline{n}^{\alpha}\left|e^{\frac{\beta}{N_{\mathrm{s}}} \sum_{\langle i, j\rangle} J_{\langle i, j\rangle}\left(a_{i}^{\dagger} a_{j}+a_{j}^{\dagger} a_{i}\right)}\right| \underline{n}^{\alpha+1}\right\rangle=\sum_{\underline{y}^{\alpha}} \prod_{\langle i, j\rangle}\left(\frac{\beta J_{\langle i, j\rangle} \sqrt{n_{j}^{\alpha+1} n_{i}^{\alpha}}}{N_{\mathrm{s}}}\right)^{y_{i \rightarrow j}^{\alpha}}\left(\frac{\beta J_{\langle i, j\rangle} \sqrt{n_{i}^{\alpha+1} n_{j}^{\alpha}}}{N_{\mathrm{s}}}\right)^{y_{j \rightarrow i}^{\alpha}} \\
\prod_{i=1}^{N} \mathbb{1}\left(n_{i}^{\alpha+1}=n_{i}^{\alpha}+\sum_{j \in \partial i}\left[y_{j \rightarrow i}^{\alpha}-y_{i \rightarrow j}^{\alpha}\right]\right)+O\left(\frac{1}{N_{\mathrm{s}}^{2}}\right),
\end{aligned}
$$

where here and in the following $\mathbb{1}(A)=1$ if the condition $A$ is fulfilled, 0 otherwise. We take by convention $x^{y=0}=1$ for any value of $x$ (including zero). The justification of (40) can be done by inspecting the behaviour of its left and right-hand-side order by order in $1 / N_{\mathrm{s}}$. The leading term corresponds to $\underline{n}^{\alpha}=\underline{n}^{\alpha+1}$, and indeed all $y$ 's must vanish at this order. At order $1 / N_{\mathrm{s}}$ a single $y$, say $y_{i \rightarrow j}^{\alpha}$, is equal to 1 , meaning that a boson has hopped from site $i$ to site $j$ between the discrete times $\alpha$ and $\alpha+1$. The term $\sqrt{n_{j}^{\alpha+1} n_{i}^{\alpha}}$ follows from the action of creation/annihilation 
operators recalled in (38). The kinetic energy part of the partition function (39) can be transformed by using the representation (40) for each time $\alpha$; reordering the various terms leads to a compact expression,

$$
Z=\lim _{N_{\mathrm{s}} \rightarrow \infty} \sum_{\underline{\boldsymbol{n}}, \underline{\boldsymbol{y}}} \prod_{\langle i, j\rangle} w_{\langle i, j\rangle}\left(\boldsymbol{y}_{\langle i, j\rangle}\right) \prod_{i=1}^{N} w_{i}\left(\boldsymbol{n}_{i},\left\{\boldsymbol{y}_{\langle i, j\rangle}\right\}_{j \in \partial i}\right) .
$$

Following the convention we already used in Sec. III bold symbols stand for time dependent quantities, with a discrete time coordinate $\alpha \in\left[1, N_{\mathrm{s}}\right]$ as long as $N_{\mathrm{s}}$ is finite, or a continuous time $\tau \in[0, \beta]$ in the limit $N_{\mathrm{s}} \rightarrow \infty$. The correspondence between the two notations is $\tau=\alpha \beta / N_{\mathrm{s}}$. We also use the shorthand $y_{\langle i, j\rangle}^{\alpha}=\left(y_{i \rightarrow j}^{\alpha}, y_{j \rightarrow i}^{\alpha}\right)$ for the pair of hopping variables on both directions of an edge $\langle i, j\rangle$. The weight of a configuration $(\underline{\boldsymbol{n}}, \underline{\boldsymbol{y}})$ factorizes into a product over all edges of

$$
w_{\langle i, j\rangle}\left(\boldsymbol{y}_{\langle i, j\rangle}\right)=\prod_{\alpha=1}^{N_{\mathrm{s}}}\left(\frac{\beta J_{\langle i, j\rangle}}{N_{\mathrm{s}}}\right)^{y_{i \rightarrow j}^{\alpha}+y_{j \rightarrow i}^{\alpha}},
$$

the hopping strength $J_{\langle i, j\rangle}$ is thus conjugated to the number of hopping events between sites $i$ and $j$. The second part of the weight in Eq. (41) is a product over the sites of

$$
\begin{aligned}
w_{i}\left(\boldsymbol{n}_{i},\left\{\boldsymbol{y}_{\langle i, j\rangle}\right\}_{j \in \partial i}\right)=\exp [ & \left.-\frac{\beta}{N_{\mathrm{s}}} \sum_{\alpha=1}^{N_{\mathrm{s}}} V_{i}\left(n_{i}^{\alpha}\right)\right] \\
& \prod_{\alpha=1}^{N_{\mathrm{s}}}\left\{\mathbb{1}\left(n_{i}^{\alpha+1}=n_{i}^{\alpha}+\sum_{j \in \partial i}\left[y_{j \rightarrow i}^{\alpha}-y_{i \rightarrow j}^{\alpha}\right]\right)\left(\sqrt{n_{i}^{\alpha+1}}\right)^{\sum_{j \in \partial i} y_{j \rightarrow i}^{\alpha}}\left(\sqrt{n_{i}^{\alpha}}\right)^{\sum_{j \in \partial i} y_{i \rightarrow j}^{\alpha}}\right\} .
\end{aligned}
$$

The first line arises directly from the local potential energy term of the Hamiltonian. The second line enforces the consistency of the occupation number trajectory $n_{i}^{\alpha}$ with the hopping events that occur on the adjacent edges, and incorporates the $\sqrt{n}$ terms arising from the action of the creation/annihilation operators.

The expression of the partition function in terms of a classical model with imaginary time-dependent variables we just obtained is valid for any underlying graph. We want to emphasize that the spatial structure of the classical model is the same as the original one: the variables $\boldsymbol{n}_{i}$ are located on the vertices of the graph, the $\boldsymbol{y}_{\langle i, j\rangle}$ on the edges, and in the weights $w_{i}$ the interactions only occur between edges and site variables which were adjacent in the original model. In particular, if the latter was locally tree-like, this remains true for the representation (41) on which the Bethe approximation can be performed, as we shall do in the next subsection.

\section{B. The Bethe free energy}

One can follow two roads to obtain the self-consistent equation (27). The first one corresponds to the reasoning presented in the simpler case of the Ising model in Sec. III A. Assuming the graph to be a tree, one can easily compute recursively $Z_{i \rightarrow j}\left(\boldsymbol{y}_{\langle i, j\rangle}\right)$, the partial partition function for the subtree rooted at $i$ excluding $j$, for a given value of the hopping trajectory $\boldsymbol{y}_{\langle i, j\rangle}$. Introducing its normalized counterpart $\eta_{i \rightarrow j}\left(\boldsymbol{y}_{\langle i, j\rangle}\right)=Z_{i \rightarrow j}\left(\boldsymbol{y}_{\langle i, j\rangle}\right) / z_{i \rightarrow j}$, one obtains

$$
\eta_{i \rightarrow j}\left(\boldsymbol{y}_{\langle i, j\rangle}\right)=\frac{1}{z_{i \rightarrow j}} w_{\langle i, j\rangle}\left(\boldsymbol{y}_{\langle i, j\rangle}\right) \sum_{\boldsymbol{n}_{i},\left\{\boldsymbol{y}_{\langle i, k\rangle}\right\}_{k \in \partial i \backslash j}} w_{i}\left(\boldsymbol{n}_{i}, \boldsymbol{y}_{\langle i, j\rangle},\left\{\boldsymbol{y}_{\langle i, k\rangle}\right\}_{k \in \partial i \backslash j}\right) \prod_{k \in \partial i \backslash j} \eta_{k \rightarrow i}\left(\boldsymbol{y}_{\langle i, k\rangle}\right) .
$$

The probability distribution of the occupation number trajectory on site $i$ is then expressed as

$$
\eta_{i}\left(\boldsymbol{n}_{i}\right)=\frac{1}{z_{i}} \sum_{\left\{\boldsymbol{y}_{\langle i, j\rangle}\right\}_{j \in \partial i}} w_{i}\left(\boldsymbol{n}_{i},\left\{\boldsymbol{y}_{\langle i, j\rangle}\right\}_{j \in \partial i}\right) \prod_{j \in \partial i} \eta_{j \rightarrow i}\left(\boldsymbol{y}_{\langle i, j\rangle}\right) .
$$

These last two equations are the analogs of Eq. (13) of the Ising model. If one considers now the homogeneous case of a Bethe lattice of connectivity $c$, with the same hopping strength $J_{\langle i, j\rangle}=J$ on all edges and $V_{i}(n)=V(n)$ for all sites, the probability laws $\eta_{i \rightarrow j}$ take a common value $\eta_{\text {cav }}$ on all directed edges, which is the solution of Eq. (27). In that equation $\boldsymbol{y}$ is an hopping trajectory on an edge, characterized more explicitly as $\boldsymbol{y}=\left(y_{+}^{1}, y_{-}^{1}, \ldots, y_{+}^{N_{\mathrm{s}}}, y_{-}^{N_{\mathrm{s}}}\right)$, where $y_{+}^{\alpha}=1$ (resp. $y_{-}^{\alpha}=1$ ) means a particle has arrived (resp. leaved) the considered site at discrete time $\alpha$. 
The expression of the weights $w_{\text {link }}$ and $w_{\text {iter }}$ of Eq. (27) is found by specializing (42) and (43) to the homogeneous situation,

$$
w_{\text {link }}(\boldsymbol{y})=\left(\frac{\beta J}{N_{\mathrm{s}}}\right)^{\sum_{\alpha=1}^{N_{\mathrm{s}}}\left[y_{+}^{\alpha}+y_{-}^{\alpha}\right]}
$$

and

$$
\begin{aligned}
w_{\text {iter }}\left(\boldsymbol{y}, \boldsymbol{y}_{1} \ldots, \boldsymbol{y}_{c-1}\right) & =\sum_{\boldsymbol{n}} e^{-\frac{\beta}{N_{\mathrm{s}}} \sum_{\alpha=1}^{N_{\mathrm{s}}} V\left(n^{\alpha}\right)} \\
& \prod_{\alpha=1}^{N_{\mathrm{s}}}\left\{\mathbb{1}\left(n^{\alpha+1}=n^{\alpha}+y_{-}^{\alpha}-y_{+}^{\alpha}+\sum_{i=1}^{c-1}\left[y_{+, i}^{\alpha}-y_{-, i}^{\alpha}\right]\right)\left(\sqrt{n^{\alpha+1}}\right)^{y_{-}^{\alpha}+\sum_{i=1}^{c-1} y_{+, i}^{\alpha}}\left(\sqrt{n^{\alpha}}\right)^{y_{+}^{\alpha}+\sum_{i=1}^{c-1} y_{-, i}^{\alpha}}\right\} .
\end{aligned}
$$

Note that the direction of $\boldsymbol{y}_{i}$, for $i \in[1, c-1]$, is reversed with respect to $\boldsymbol{y}$, hence the inversion of the signs on the variation of $n^{\alpha}$ from one discrete time to the next. Finally the weight $w_{\text {site }}$ used in Eq. (32) to obtain the probability distribution of an occupation number trajectory reads

$$
w_{\text {site }}\left(\boldsymbol{n}, \boldsymbol{y}_{1} \ldots, \boldsymbol{y}_{c}\right)=e^{-\frac{\beta}{N_{\mathrm{s}}} \sum_{\alpha=1}^{N_{\mathrm{s}}} V\left(n^{\alpha}\right)} \prod_{\alpha=1}^{N_{\mathrm{s}}}\left\{\mathbb{1}\left(n^{\alpha+1}=n^{\alpha}+\sum_{i=1}^{c}\left[y_{+, i}^{\alpha}-y_{-, i}^{\alpha}\right]\right)\left(\sqrt{n^{\alpha+1}}\right)^{\sum_{i=1}^{c} y_{+, i}^{\alpha}}\left(\sqrt{n^{\alpha}}\right) \sum_{i=1}^{c} y_{-, i}^{\alpha}\right\} .
$$

The second strategy to reach the equation (27) on $\eta_{\text {cav }}$ consists in first writing the Bethe approximation [39] for the free-energy of the model defined in (41) for an arbitrary graph. This yields

$$
\begin{aligned}
F=-\frac{1}{\beta} \ln Z= & -\frac{1}{\beta} \sum_{i=1}^{N} \ln \left(\sum_{\boldsymbol{n}_{i},\left\{\boldsymbol{y}_{\langle i, j\rangle}\right\}_{j \in \partial i}} w_{i}\left(\boldsymbol{n}_{i},\left\{\boldsymbol{y}_{\langle i, j\rangle}\right\}_{j \in \partial i}\right) \prod_{j \in \partial i} \eta_{j \rightarrow i}\left(\boldsymbol{y}_{\langle i, j\rangle}\right)\right) \\
& +\frac{1}{\beta} \sum_{\langle i, j\rangle} \ln \left(\sum_{\boldsymbol{y}_{\langle i, j\rangle}} \frac{1}{w_{\langle i, j\rangle}\left(\boldsymbol{y}_{\langle i, j\rangle}\right)} \eta_{i \rightarrow j}\left(\boldsymbol{y}_{\langle i, j\rangle}\right) \eta_{j \rightarrow i}\left(\boldsymbol{y}_{\langle i, j\rangle}\right)\right) .
\end{aligned}
$$

One can check that this relation is exact whenever the graph is a tree, otherwise it corresponds to the Bethe approximation. An important property of this expression of the free-energy is its variational character: the stationarity conditions with respect to the parameters $\eta_{i \rightarrow j}$ are nothing but the local recurrence equations (44). This means that the computation of the derivatives of the free-energy with respect to its parameters $\mu, \beta, J_{\langle i, j\rangle}, \ldots$ can be performed by considering only the explicit dependence on these parameters, discarding the implicit dependence through the $\eta_{i \rightarrow j}$. We shall also show in Appendix A how to devise a further (static) approximation based on this variational expression of the free-energy. Considering the particular case of an homogeneous Bethe lattice leads to the following free-energy per site:

$$
f=\lim _{N \rightarrow \infty} \frac{-1}{\beta N} \ln Z=-\frac{1}{\beta} \ln \left(\sum_{\boldsymbol{n}, \boldsymbol{y}_{1}, \ldots, \boldsymbol{y}_{c}} w_{\text {site }}\left(\boldsymbol{n}, \boldsymbol{y}_{1}, \ldots, \boldsymbol{y}_{c}\right) \eta_{\text {cav }}\left(\boldsymbol{y}_{1}\right) \ldots \eta_{\text {cav }}\left(\boldsymbol{y}_{c}\right)\right)+\frac{c}{2 \beta} \ln \left(\sum_{\boldsymbol{y}} \frac{1}{w_{\text {link }}(\boldsymbol{y})} \eta_{\text {cav }}^{2}(\boldsymbol{y})\right)
$$

where we have used the fact that a regular graph of $N$ vertices and connectivity $c$ has $c N / 2$ edges. One can see that (27) is the stationarity condition of (50) with respect to $\eta_{\text {cav }}$.

By using the recurrence equations (44) it is possible to rewrite the Bethe approximation of the free-energy, for an arbitrary graph, as

$$
F=-\frac{1}{\beta} \ln Z=-\frac{1}{\beta} \sum_{\langle i, j\rangle} \ln \left(\sqrt{z_{i \rightarrow j} z_{j \rightarrow i}}\right)+\frac{1}{\beta} \sum_{i=1}^{N} \frac{c_{i}-2}{2} \ln z_{i},
$$

where $c_{i}$ is the degree of vertex $i$, and the various $z$ 's correspond to the normalizations in (44),(45). This form does not have the variational property explained above; it has however the advantage of being easier to compute numerically. In particular for the homogeneous case it yields

$$
f=\lim _{N \rightarrow \infty} \frac{-1}{\beta N} \ln Z=-\frac{c}{2 \beta} \ln z_{\text {cav }}+\frac{c-2}{2 \beta} \ln z_{\text {site }},
$$

where $z_{\text {cav }}$ and $z_{\text {site }}$ are the normalizations in, respectively, Eqs. (27) and (32). 


\section{The resolution of the cavity equation}

As we have explained in Sec. IV A we use a weighted sample representation of $\eta_{\text {cav }}$ to solve (27). The point we want to specify more precisely here is the method used to sample hopping trajectories from the conditional law $P\left(\boldsymbol{y} \mid \boldsymbol{y}_{1}, \ldots, \boldsymbol{y}_{c-1}\right)$ and to compute the associated normalization $\mathcal{Z}\left(\boldsymbol{y}_{1}, \ldots, \boldsymbol{y}_{c-1}\right)$ (both defined in Eq. (31)). A similar construction can be found for the slightly simpler case of quantum spin $1 / 2$ models in [34].

Let us begin with the computation of $\mathcal{Z}$. A first important remark, already mentioned above, is that in the continuous time limit $\left(N_{\mathrm{s}} \rightarrow \infty\right)$ the hopping trajectories $\boldsymbol{y}$ typically contain only a finite number (with respect to $N_{\mathrm{s}}$ ) of hopping events, i.e. of discrete times $\alpha$ where $y_{ \pm}^{\alpha} \neq 0$. We can thus assume without loss of generality that the hopping events for the different trajectories occur at different values of the discrete time. Let us call $p$ the total number of hopping events occuring in $\left(\boldsymbol{y}_{1}, \ldots, \boldsymbol{y}_{c-1}\right)$, and denote $\alpha_{1}<\cdots<\alpha_{p}$ their discrete time of occurence. We consider the Hilbert space of a single site, with $a$ and $a^{\dagger}$ the annihilation/creation operators, and call $b_{j}=a($ resp. $b_{j}=a^{\dagger}$ ) if the hopping of time $\alpha_{j}$ is towards (resp. outside) the vertex under consideration. Finally we define $c_{\alpha}=b_{j}$ when $\alpha=\alpha_{j}, c_{\alpha}=\mathbb{1}$ (the identity operator) otherwise. A moment of thought reveals that

$$
\mathcal{Z}\left(\boldsymbol{y}_{1}, \ldots, \boldsymbol{y}_{c-1}\right)=\sum_{\boldsymbol{y}} w_{\text {link }}(\boldsymbol{y}) w_{\text {iter }}\left(\boldsymbol{y}, \boldsymbol{y}_{1}, \ldots, \boldsymbol{y}_{c-1}\right)=\sum_{\boldsymbol{n}} \prod_{\alpha=1}^{N_{\mathrm{s}}}\left\langle n^{\alpha}\left|e^{\frac{\beta}{N_{\mathrm{s}}}\left(-V\left(a^{\dagger} a\right)+J\left(a+a^{\dagger}\right)\right)} c_{\alpha}\right| n^{\alpha+1}\right\rangle
$$

up to corrections of order $N_{\mathrm{s}}^{-2}$. The sum over $\boldsymbol{n}$ can thus be written as a trace of this product of operators. To perform the continuous time limit it is convenient to define $\tau_{j}=\frac{\beta}{N_{\mathrm{s}}} \alpha_{j}$, which are the continuous times of the hopping events in $\left(\boldsymbol{y}_{1}, \ldots, \boldsymbol{y}_{c-1}\right)$. We also introduce $\widehat{W}(\lambda)=e^{\lambda\left(-V\left(a^{\dagger} a\right)+J\left(a+a^{\dagger}\right)\right)}$, the propagator of an imaginary time evolution on an interval of length $\lambda$ for a single site Hamiltonian $V\left(a^{\dagger} a\right)-J\left(a+a^{\dagger}\right)$. We thus obtain finally

$$
\mathcal{Z}\left(\boldsymbol{y}_{1}, \ldots, \boldsymbol{y}_{c-1}\right)=\operatorname{Tr}\left(\widehat{W}\left(\tau_{1}\right) b_{1} \widehat{W}\left(\tau_{2}-\tau_{1}\right) b_{2} \ldots \widehat{W}\left(\tau_{p}-\tau_{p-1}\right) b_{p} \widehat{W}\left(\beta-\tau_{p}\right)\right)
$$

The single site Hilbert space is a priori infinite dimensional because the number of particles is not bounded. Very high occupation numbers are however unlikely because of their prohibitive potential energy. We can thus safely put a cutoff $N_{\text {cut }}$ on the possible values of $n$, which will not change the properties of the model if $N_{\text {cut }}$ is sufficiently large with respect to the average density of particles. This would amount formally to take $V(n)=+\infty$ for $n \geq N_{\text {cut }}$. In this case the dimension of the Hilbert space is reduced to $N_{\text {cut }}$, and (54) is nothing but the trace of the product of a finite number of finite size matrices, whose numerical evaluation does not present any particular difficulty.

We turn now to the problem of the generation of an hopping trajectory $\boldsymbol{y}$ given the ones of the $c-1$ other neighbors, according to the law $P\left(\boldsymbol{y} \mid \boldsymbol{y}_{1}, \ldots, \boldsymbol{y}_{c-1}\right)$ stated in (31). As explained in Sec. IV A (see in particular Fig. 5), we can determine $\boldsymbol{y}$ by first drawing the occupation number trajectory $\boldsymbol{n}$ and then deduce $\boldsymbol{y}$ from its discontinuities not associated to hopping events in $\left(\boldsymbol{y}_{1}, \ldots, \boldsymbol{y}_{c-1}\right)$. We stick to the notation $\tau_{1}<\ldots<\tau_{p}$ and $b_{1}, \ldots, b_{p}$ for the parametrization of the hopping events in $\left(\boldsymbol{y}_{1}, \ldots, \boldsymbol{y}_{c-1}\right)$. Let us call $n_{0}=n(\tau=0), n_{i}\left(\right.$ resp. $\left.n_{i}^{\prime}\right)$ the value of $n(\tau)$ at a time just after $\tau_{i}$ (resp. just before $\tau_{i+1}$ ), with the conventions $n_{p}^{\prime}=n_{0}$. The joint probability law of these occupation numbers which arise from the expressions of $w_{\text {link }}$ and $w_{\text {iter }}$ given in Eqs. $(46)$, (47) reads in the continuous time limit

$$
P\left(n_{0}, n_{0}^{\prime}, n_{1}, n_{1}^{\prime}, \ldots, n_{p} \mid \boldsymbol{y}_{1}, \ldots, \boldsymbol{y}_{c-1}\right)=\frac{1}{\mathcal{Z}\left(\boldsymbol{y}_{1}, \ldots, \boldsymbol{y}_{c-1}\right)}\left\langle n_{0}\left|\widehat{W}\left(\tau_{1}\right)\right| n_{0}^{\prime}\right\rangle \prod_{i=1}^{p}\left\{\left\langle n_{i-1}^{\prime}\left|b_{i}\right| n_{i}\right\rangle\left\langle n_{i}\left|\widehat{W}\left(\tau_{i+1}-\tau_{i}\right)\right| n_{i}^{\prime}\right\rangle\right\}
$$

with $\tau_{p+1}=\beta$. This probability law is well normalized according to the above expression of $\mathcal{Z}\left(\boldsymbol{y}_{1}, \ldots, \boldsymbol{y}_{c-1}\right)$. Moreover because of the "unidimensional" structure of the imaginary time axis it is rather simple to generate a set of integer numbers $\left(n_{0}, n_{0}^{\prime}, n_{1}, n_{1}^{\prime}, \ldots, n_{p}\right)$ according to this law. Once these intermediate occupation numbers are known, the generation of the occupation trajectory $n(\tau)$ can be done independently on each of the $p+1$ time intervals between the imposed hopping events. On the $i+1$ 'th interval between $\tau_{i}$ and $\tau_{i+1}$ one has to generate the trajectory corresponding to a path integral representation of the effective Hamiltonian $V\left(a^{\dagger} a\right)-J\left(a+a^{\dagger}\right)$, conditioned to begin in $n_{i}$ at time $\tau_{i}$ and to end in $n_{i}^{\prime}$ at $\tau_{i+1}$.

To simplify the notation let us consider this problem for an interval of imaginary time $[0, \lambda]$, with boundary conditions $n(0)=n, n(\lambda)=n^{\prime}$. One way to justify the sampling procedure is to start with the identity

$$
e^{\lambda(X+Y)}=e^{\lambda X}+\int_{0}^{\lambda} \mathrm{d} \tau e^{\tau X} Y e^{(\lambda-\tau)(X+Y)}
$$


valid for any non-commutative operators $X$ and $Y$. We apply it with $X=-V\left(a^{\dagger} a\right)$ and $Y=J\left(a+a^{\dagger}\right)$, which yields

$$
\begin{aligned}
\left\langle n|\widehat{W}(\lambda)| n^{\prime}\right\rangle & =\left\langle n\left|e^{-\lambda V\left(a^{\dagger} a\right)}+\int_{0}^{\lambda} \mathrm{d} \tau e^{-\tau V\left(a^{\dagger} a\right)} J\left(a+a^{\dagger}\right) \widehat{W}(\lambda-\tau)\right| n^{\prime}\right\rangle \\
& =\delta_{n, n^{\prime}} e^{-\lambda V(n)}+J \int_{0}^{\lambda} \mathrm{d} \tau e^{-\tau V(n)}\left\langle n\left|\left(a+a^{\dagger}\right) \widehat{W}(\lambda-\tau)\right| n^{\prime}\right\rangle .
\end{aligned}
$$

The interpretation of this formula is as follows: a path representative of the matrix element $\left\langle n|\widehat{W}(\lambda)| n^{\prime}\right\rangle$ is either a constant trajectory (possible only if $n=n^{\prime}$ ), or it is constant up to a time $\tau$, where it jumps to $n \pm 1$, and is followed by a path of length $\lambda-\tau$ with boundary conditions $n \pm 1$ and $n^{\prime}$. We can thus sample the path $n(\tau)$ according to the following recursive procedure:

- if $n=n^{\prime}$ and with probability $e^{-\lambda V(n)} /\langle n|\widehat{W}(\lambda)| n\rangle$, set $n(\tau)=n$ for $\tau \in[0, \beta]$ and exit the procedure

- otherwise

- draw a time $\tau \in[0, \lambda]$ with the probability law written below in Eq. (59)

- draw $\sigma=1$ with probability $\frac{\left\langle n|a \widehat{W}(\lambda-\tau)| n^{\prime}\right\rangle}{\left\langle n\left|\left(a+a^{\dagger}\right) \widehat{W}(\lambda-\tau)\right| n^{\prime}\right\rangle}, \sigma=-1$ otherwise

- set $n\left(\tau^{\prime}\right)=n$ for $\tau^{\prime} \in[0, \tau]$

- call the same procedure with initial condition $n+\sigma$, final condition $n^{\prime}$, for the imaginary time interval $[\tau, \lambda]$

The probability law for the time $\tau$ of the first discontinuity in the number occupation trajectory follows from (58); it is more convenient to express it as the probability $G(u)$ that $\tau \leq u$,

$$
G(u)=\frac{\int_{0}^{u} \mathrm{~d} \tau e^{-\tau V(n)}\left\langle n\left|\left(a+a^{\dagger}\right) \widehat{W}(\lambda-\tau)\right| n^{\prime}\right\rangle}{\int_{0}^{\lambda} \mathrm{d} \tau e^{-\tau V(n)}\left\langle n\left|\left(a+a^{\dagger}\right) \widehat{W}(\lambda-\tau)\right| n^{\prime}\right\rangle}
$$

Because of the cutoff on the possible number occupation one can numerically compute this function by diagonalizing the effective Hamiltonian (only once for each choice of the parameters $U, \mu, J$ ). Drawing $\tau$ then amounts to use a random number $G$ uniformly in $[0,1]$ and set $\tau=u^{-1}(G)$ (the functional inverse of $G(u)$ ).

\section{The computation of the observables}

We will show now that, as announced in Sec. IV A, all the physical observables can be obtained as averages over $\eta_{\text {cav }}$ of some functions of the hopping trajectories. The computation of such an average can then be done as a simple sampling of the population representing $\eta_{\text {cav }}$, as explained in Eq. (34).

Let us begin with the free-energy. Using the formula (52) its computation amounts to the determination of the normalization constants $z_{\text {cav }}$ and $z_{\text {site }}$. The former reads

$$
z_{\mathrm{cav}}=\sum_{\boldsymbol{y}_{1}, \ldots, \boldsymbol{y}_{c-1}} \eta_{\mathrm{cav}}\left(\boldsymbol{y}_{1}\right) \ldots \eta_{\mathrm{cav}}\left(\boldsymbol{y}_{c-1}\right) \mathcal{Z}\left(\boldsymbol{y}_{1}, \ldots, \boldsymbol{y}_{c-1}\right)
$$

which is readily evaluated since we obtained an explicit form of $\mathcal{Z}\left(\boldsymbol{y}_{1}, \ldots, \boldsymbol{y}_{c-1}\right)$ in Eq. (54). The latter is seen from (32) to be

$$
z_{\text {site }}=\sum_{\boldsymbol{y}_{1}, \ldots, \boldsymbol{y}_{c}} \eta_{\mathrm{cav}}\left(\boldsymbol{y}_{1}\right) \ldots \eta_{\mathrm{cav}}\left(\boldsymbol{y}_{c}\right) \sum_{\boldsymbol{n}} w_{\text {site }}\left(\boldsymbol{n}, \boldsymbol{y}_{1}, \ldots, \boldsymbol{y}_{c}\right)
$$

Using again the notation $\tau_{1}<\cdots<\tau_{p}$ and $b_{1}, \ldots, b_{p}$ for the parametrization of the hopping events in $\left(\boldsymbol{y}_{1}, \ldots, \boldsymbol{y}_{c}\right)$ and exploiting the expression (48) for $w_{\text {site }}$, we obtain in the continuous-time limit

$$
z_{\text {site }}=\sum_{\boldsymbol{y}_{1}, \ldots, \boldsymbol{y}_{c}} \eta_{\text {cav }}\left(\boldsymbol{y}_{1}\right) \ldots \eta_{\text {cav }}\left(\boldsymbol{y}_{c}\right) \operatorname{Tr}\left(\widehat{W}_{0}\left(\tau_{1}\right) b_{1} \widehat{W}_{0}\left(\tau_{2}-\tau_{1}\right) b_{2} \ldots b_{p} \widehat{W}_{0}\left(\beta-\tau_{p}\right)\right)
$$

where we defined $\widehat{W}_{0}(\lambda)=e^{-\lambda V\left(a^{\dagger} a\right)}$, the single-site propagator without its hopping term. Indeed all the possible hopping events to and from the considered site are fixed by the $c$ trajectories $\boldsymbol{y}_{1}, \ldots, \boldsymbol{y}_{c}$. This trace is over a product 
of finite size matrices (thanks to the bound $N_{\text {cut }}$ on the occupation numbers) and hence computationally harmless. Note that only configurations of $\boldsymbol{y}_{1}, \ldots, \boldsymbol{y}_{c}$ which encode the same number of jumps towards/outside the central site do contribute, because $\widehat{W}_{0}$ is diagonal in the number basis.

We consider now the thermal average $\left\langle q\left(a_{i}^{\dagger} a_{i}\right)\right\rangle$ of an arbitrary function $q(n)$ of the number operator on one site. From the expression (32) of the probability distribution $\eta(\boldsymbol{n})$ we obtain

$$
\left\langle q\left(a_{i}^{\dagger} a_{i}\right)\right\rangle=\frac{1}{z_{\text {site }}} \sum_{\boldsymbol{y}_{1}, \ldots, \boldsymbol{y}_{c}} \eta_{\text {cav }}\left(\boldsymbol{y}_{1}\right) \ldots \eta_{\text {cav }}\left(\boldsymbol{y}_{c}\right) \operatorname{Tr}\left(q\left(a^{\dagger} a\right) \widehat{W}_{0}\left(\tau_{1}\right) b_{1} \widehat{W}_{0}\left(\tau_{2}-\tau_{1}\right) b_{2} \ldots b_{p} \widehat{W}_{0}\left(\beta-\tau_{p}\right)\right)
$$

This formula allows to compute the density of particles, the average local energy $e_{L}$ and the occupation probability $P_{n_{0}}$ by taking $q(n)=n, q(n)=V(n)$ and $q(n)=\delta_{n, n_{0}}$, respectively.

The order parameter $\left\langle a_{i}\right\rangle$ is obtained by the insertion of an annihilation operator in the effective single-site problem,

$$
\left\langle a_{i}\right\rangle=\frac{1}{z_{\text {site }}} \sum_{\boldsymbol{y}_{1}, \ldots, \boldsymbol{y}_{c}} \eta_{\text {cav }}\left(\boldsymbol{y}_{1}\right) \ldots \eta_{\text {cav }}\left(\boldsymbol{y}_{c}\right) \operatorname{Tr}\left(a \widehat{W}_{0}\left(\tau_{1}\right) b_{1} \widehat{W}_{0}\left(\tau_{2}-\tau_{1}\right) b_{2} \ldots b_{p} \widehat{W}_{0}\left(\beta-\tau_{p}\right)\right)
$$

One has indeed by definition $\left\langle a_{i}\right\rangle=\operatorname{Tr}\left[a_{i} e^{-\beta H}\right] / Z$, where the trace is here over the Hilbert space of the $N$-sites Hamiltonian. One can reproduce all the steps leading to the representation (41) of the partition function, with the additional operator $a_{i}$ modifying the expression of the weight $w_{i}$ on the considered site. The other sites are however left unmodified, and hence integrating over them leads to the same equation on $\eta_{\text {cav }}$. The presence of $a_{i}$ only shows up when the sum over the degrees of freedom of site $i$ is performed, and leads to this insertion of the annihilation operator in the single-site computation (64). We now come back on the problem of the initial condition in the population dynamics mentioned in Sec. IV A. One can indeed see that (64) strictly vanishes whenever all hopping trajectories in the support of $\eta_{\text {cav }}$ have the same number of jumps in the two directions of their edge. Moreover this symmetry is conserved by the iterative equation (27); we thus had to initialize the population dynamics algorithm including asymmetric hopping trajectories. In the "insulating" phase these trajectories disappear during the iterations, while in the "superfluid" a finite fraction of them keeps the asymmetry, thus allowing for a non zero value of $\left\langle a_{i}\right\rangle$.

The computation of the Green function $G_{>}^{i}(\tau)=\operatorname{Tr}\left[e^{-(\beta-\tau) H} a_{i} e^{-\tau H} a_{i}^{\dagger}\right] / Z$ can be done similarly, with now the insertion of two creation/annihilation operators in the single-site problem. Similarly to Eq. (63), and using the same notations, one obtains

$$
\begin{aligned}
G_{>}(\tau)= & \frac{1}{z_{\text {site }}} \sum_{\boldsymbol{y}_{1}, \ldots, \boldsymbol{y}_{c}} \eta_{\text {cav }}\left(\boldsymbol{y}_{1}\right) \ldots \eta_{\text {cav }}\left(\boldsymbol{y}_{c}\right) \times \\
& \times \operatorname{Tr}\left(a \widehat{W}_{0}\left(\tau_{1}\right) b_{1} \widehat{W}_{0}\left(\tau_{2}-\tau_{1}\right) b_{2} \ldots b_{i} \widehat{W}_{0}\left(\tau-\tau_{i}\right) a^{\dagger} \widehat{W}_{0}\left(\tau_{i+1}-\tau\right) b_{i+1} \cdots b_{p} \widehat{W}_{0}\left(\beta-\tau_{p}\right)\right)
\end{aligned}
$$

where the index $i$ is such that $\tau_{i}<\tau<\tau_{i+1}$.

We turn now to the computation of the average kinetic energy $e_{K}=\left\langle H_{K}\right\rangle / N$. By definition it is equal to the derivative of the free-energy per site with respect to $J$. Using the variational property of the expression (50), we can thus write

$$
e_{K}=-\frac{c}{2 \beta J} \frac{\sum_{\boldsymbol{y}} \frac{1}{w_{\text {link }}(\boldsymbol{y})} \eta_{\text {cav }}^{2}(\boldsymbol{y})|\boldsymbol{y}|}{\sum_{\boldsymbol{y}} \frac{1}{w_{\text {link }}(\boldsymbol{y})} \eta_{\text {cav }}^{2}(\boldsymbol{y})}
$$

where we have defined $|\boldsymbol{y}|$ as the total number of hopping events in $\boldsymbol{y}$. Indeed the only explicit dependence on $J$ in (50) is in the weight $w_{\text {link }}$ given in Eq. (46). Replacing one of the $\eta_{\text {cav }}$ by its expression (27) both in the numerator and the denominator, and reordering terms leads to

$$
e_{K}=-\frac{c}{2 \beta J} \frac{1}{z_{\text {site }}} \sum_{\boldsymbol{y}_{1}, \ldots, \boldsymbol{y}_{c}} \eta_{\text {cav }}\left(\boldsymbol{y}_{1}\right) \ldots \eta_{\text {cav }}\left(\boldsymbol{y}_{c}\right)\left|\boldsymbol{y}_{1}\right| \operatorname{Tr}\left(\widehat{W}_{0}\left(\tau_{1}\right) b_{1} \widehat{W}_{0}\left(\tau_{2}-\tau_{1}\right) b_{2} \ldots b_{p} \widehat{W}_{0}\left(\beta-\tau_{p}\right)\right)
$$

Finally we consider the computation of the one-particle density matrix $\rho_{d}$ for two sites at distance $d$. For notational simplicity we shall call them 0 and $d, \rho_{d}=\left\langle a_{0}^{\dagger} a_{d}\right\rangle$. The unique path linking these two sites is schematized in Fig. 13; we have distinguished the hopping trajectories $\boldsymbol{y}_{i}$ along the chain for $i \in[0, d-1]$, from the contribution of the edges outside the chain, $\boldsymbol{y}_{i, j}^{\prime}$. The index $j$ runs from 1 to $c_{i}$, with $c_{0}=c_{d}=c-1$ and $c_{1}=\cdots=c_{d-1}=c-2$, 


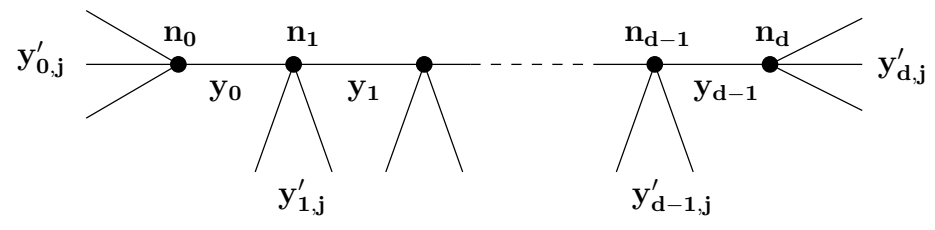

FIG. 13: Computation of the one-particle density matrix at distance $d, \rho_{d}=\left\langle a_{0}^{\dagger} a_{d}\right\rangle$.

as the extremities 0 and $d$ have one less neighbor in the chain. The computation of $\left\langle a_{0}^{\dagger} a_{d}\right\rangle$ is a generalization of the determination of $\left\langle a_{i}\right\rangle$ explained above: one writes $\left\langle a_{0}^{\dagger} a_{d}\right\rangle=\operatorname{Tr}\left[a_{0}^{\dagger} a_{d} e^{-\beta H}\right] / Z$ and expresses the numerator with the Suzuki-Trotter formula where the local weights $w_{0}$ (resp. $w_{d}$ are modified with the insertion of a creation (resp. annihilation) operator. Summing over all degrees of freedom outside the chain of sites between 0 and $d$ leads to

$$
\left\langle a_{0}^{\dagger} a_{d}\right\rangle=\frac{\sum_{\left\{\boldsymbol{y}_{i, j}^{\prime}\right\} i, j} \prod_{\text {cav }}\left(\boldsymbol{y}_{i, j}^{\prime}\right) \sum_{\left\{\boldsymbol{y}_{i}\right\}} \prod_{i} w_{\text {link }}\left(\boldsymbol{y}_{i}\right) \sum_{\left\{\boldsymbol{n}_{i}\right\}} w_{\text {site }}^{+}\left(\boldsymbol{n}_{0}, \boldsymbol{y}_{0},\left\{\boldsymbol{y}_{0, j}^{\prime}\right\}\right) \prod_{i} w_{\text {site }}\left(\boldsymbol{n}_{i}, \boldsymbol{y}_{i-1}, \boldsymbol{y}_{i},\left\{\boldsymbol{y}_{i, j}^{\prime}\right\}\right) w_{\text {site }}^{-}\left(\boldsymbol{n}_{d}, \boldsymbol{y}_{d-1},\left\{\boldsymbol{y}_{d, j}^{\prime}\right\}\right)}{\sum_{\left\{\boldsymbol{y}_{i, j}^{\prime}\right\} i, j} \prod_{\text {cav }}\left(\boldsymbol{y}_{i, j}^{\prime}\right) \sum_{\left\{\boldsymbol{y}_{i}\right\}} \prod_{i} w_{\text {link }}\left(\boldsymbol{y}_{i}\right) \sum_{\left\{\boldsymbol{n}_{i}\right\}} w_{\text {site }}\left(\boldsymbol{n}_{0}, \boldsymbol{y}_{0},\left\{\boldsymbol{y}_{0, j}^{\prime}\right\}\right) \prod_{i} w_{\text {site }}\left(\boldsymbol{n}_{i}, \boldsymbol{y}_{i-1}, \boldsymbol{y}_{i},\left\{\boldsymbol{y}_{i, j}^{\prime}\right\}\right) w_{\text {site }}\left(\boldsymbol{n}_{d}, \boldsymbol{y}_{d-1},\left\{\boldsymbol{y}_{d, j}^{\prime}\right\}\right)},
$$

where in the numerator $w^{ \pm}$are modifications of the vertex weight (48) due to the insertion of the creation/annihilation operators on sites 0 and $d$. Integrating progressively the degrees of freedom along the chain one can easily shows that the denominator of (68) equals $z_{\text {cav }}^{d} z_{\text {site }}$. To compute the numerator we shall define a sequence of probability distributions $\eta_{\text {cav }}^{(i)}$, as the law of the hopping trajectories $\boldsymbol{y}_{i}$ along a chain where the part between $i+1$ and $d$ is removed. This can be computed by recurrence on $i$, with

$$
\eta_{\text {cav }}^{(0)}(\boldsymbol{y})=\frac{1}{z_{\text {cav }}^{(0)}} w_{\text {link }}(\boldsymbol{y}) \sum_{\boldsymbol{y}_{1}, \ldots, \boldsymbol{y}_{c-1}} \eta_{\text {cav }}\left(\boldsymbol{y}_{1}\right) \ldots \eta_{\mathrm{cav}}\left(\boldsymbol{y}_{c-1}\right) \sum_{n} w_{\mathrm{site}}^{+}\left(\boldsymbol{n}, \boldsymbol{y}, \boldsymbol{y}_{1}, \ldots, \boldsymbol{y}_{c-1}\right)
$$

and

$$
\eta_{\mathrm{cav}}^{(i+1)}(\boldsymbol{y})=\frac{1}{z_{\mathrm{cav}}^{(i+1)}} w_{\mathrm{link}}(\boldsymbol{y}) \sum_{\boldsymbol{y}_{1}, \ldots, \boldsymbol{y}_{c-1}} \eta_{\mathrm{cav}}^{(i)}\left(\boldsymbol{y}_{1}\right) \eta_{\mathrm{cav}}\left(\boldsymbol{y}_{2}\right) \ldots \eta_{\mathrm{cav}}\left(\boldsymbol{y}_{c-1}\right) \sum_{\boldsymbol{n}} w_{\mathrm{site}}\left(\boldsymbol{n}, \boldsymbol{y}, \boldsymbol{y}_{1}, \ldots, \boldsymbol{y}_{c-1}\right)
$$

These probability distributions can be encoded numerically as weighted samples, in the same way as we did for $\eta_{\text {cav }}$. Once they have been determined up to distance $d-1$ we can compute

$$
z_{\text {site }}^{(d)}=\sum_{\boldsymbol{y}_{1}, \ldots, \boldsymbol{y}_{c}} \eta_{\text {cav }}^{(d-1)}\left(\boldsymbol{y}_{1}\right) \eta_{\text {cav }}\left(\boldsymbol{y}_{2}\right) \ldots \eta_{\text {cav }}\left(\boldsymbol{y}_{c}\right) \sum_{\boldsymbol{n}} w_{\text {site }}^{-}\left(\boldsymbol{n}, \boldsymbol{y}_{1}, \ldots, \boldsymbol{y}_{c}\right)
$$

One then finds

$$
\left\langle a_{0}^{\dagger} a_{d}\right\rangle=\frac{z_{\text {site }}^{(d)}}{z_{\text {site }}^{d-1}} \prod_{i=0}^{d-1} \frac{z_{\text {cav }}^{(i)}}{z_{\text {cav }}}
$$

\section{CONCLUSIONS}

In this paper we have extended the analytical tools of the quantum cavity method to Bethe lattice bosonic models. The cavity approach yields a self-consistent equation for the probability of hopping trajectories in continuous imaginary time, which can be efficiently solved numerically using the population dynamics algorithm. Though this numerical step is unavoidable to obtain quantitative predictions it is distinct from a Quantum Monte Carlo algorithm, in particular the thermodynamic limit is taken analytically.

We have shown how this method allows to compute easily several physical observables such as thermodynamic quantities (average occupation number, energy, free energy, condensate fraction, ... ) as well as imaginary time Green 
functions. Let us emphasize in particular that computing the free-energy (which is often difficult with other methods, requiring for instance thermodynamic integration in Monte Carlo simulations) is crucial to determine the location of first-order phase transitions which can occur in generalized versions of the model.

The finite connectivity of the Bethe lattice leads to two kind of improvements with respect to the mean-field description: a distance between two sites can be defined, which allows the computation of spatial correlation functions. Moreover the description of the Mott Insulator phase is richer, with a non-trivial effect of the hopping of the particles. The prediction for the zero-temperature phase diagram of Bethe lattices with connectivity 4 and 6 is in reasonable agreement with the Quantum Monte Carlo simulations in 2 and 3 dimensions [17, 18].

Let us sketch some possible directions for future work. A first possibility would be to explore the next levels of the Cluster Variation Method [26] hierarchy of approximations, which contains the mean-field and the Bethe approximations as its first two steps. This approach, similar in spirit to the Cluster DMFT [50], should improve the accuracy of the predictions for small dimensions. It should also be interesting to investigate the effect of disorder in the Bethe lattice model; if the Bose Glass phase $[1,27]$ is destroyed at the mean-field level, it could be possible to describe it on the Bethe lattice, which can indeed display localization properties [28]. Another open direction is the study of several generalizations of the Bose-Hubbard model, including bosons with spin, multiple interacting species of particles [23, 24, 51, 52], and interactions between nearest-neighbor sites [53]. In particular in the latter case, we expect that the presence of interactions inducing geometrical frustration will give rise to glass phases, since the same happens in the classical limit of zero hopping [54]. It would be very interesting to check whether these glass phases could exhibit Bose-Einstein Condensation as it happens for their crystalline counterparts [55]. This would add some new element to the ongoing discussion on the relevance of disorder to induce superfluidity [56], and might be important to interpret and understand recent experiments on supersolidity [57].

We finally point out that, although in this paper we have only focused on the very low temperature regime (except for the transition temperature at unit filling displayed in Fig. 9), the method presented here can be applied at higher temperature (and is even numerically easier in that case), which can be useful for the interpretation of cold atoms experiments [58]. From this perspective the case of unidimensional systems should deserve a special attention, as they fall in the category of tree structure and could thus be solved with the cavity method, even in the presence of a site-dependent chemical potential modelizing the trapping field.

\section{Acknowledgments}

The method we used in this paper has been first developed for quantum spins in collaboration with F. Krzakala and A. Rosso [34] whom we warmly thank. We also acknowledge useful comments on a preliminary version of the manuscript from G. Biroli, K. Byczuk, G. Carleo, N. Dupuis, J. Estève, F. Gerbier, D. Vollhardt and M. Schirò, and M. Mézard and R. Thomale for interesting discussions.

\section{APPENDIX A: STATIC APPROXIMATION}

We have obtained in (49) a variational expression of the Bethe free-energy, in terms of the parameters $\eta_{i \rightarrow j}\left(\boldsymbol{y}_{\langle i, j\rangle}\right)$. A further approximation consists in restricting the $\eta_{i \rightarrow j}$ to a simply parametrized subspace, and finding the extremum of (49) within this simpler ansatz. A possible choice for this parametrization, inspired by the so-called static approximation developed for quantum spin models [34, 59], consists in retaining in $\boldsymbol{y}_{\langle i, j\rangle}$ only the information on the number and direction of the jumps, but assuming their times of occurence to be uniformly distributed. This corresponds in formula to the ansatz

$$
\eta_{i \rightarrow j}\left(\boldsymbol{y}_{\langle i, j\rangle}\right)=\sum_{n_{i \rightarrow j}, n_{j \rightarrow i}=0}^{\infty} \rho_{i \rightarrow j}\left(n_{i \rightarrow j}, n_{j \rightarrow i}\right) \frac{1}{\left(\begin{array}{c}
N_{\mathrm{s}} \\
n_{i \rightarrow j}
\end{array}\right)\left(\begin{array}{c}
N_{\mathrm{s}} \\
n_{j \rightarrow i}
\end{array}\right)} \mathbb{1}\left(\sum_{\alpha} y_{i \rightarrow j}^{\alpha}=n_{i \rightarrow j}\right) \mathbb{1}\left(\sum_{\alpha} y_{j \rightarrow i}^{\alpha}=n_{j \rightarrow i}\right),
$$

where $\rho_{i \rightarrow j}$ is the distribution probability for the number of jumps from $i$ to $j$ and viceversa. The two terms of the variational free-energy (49) can be computed within this ansatz. One obtains indeed in the limit $N_{\mathrm{s}} \rightarrow \infty$ :

$$
\sum_{\boldsymbol{y}_{\langle i, j\rangle}} \frac{1}{w_{\langle i, j\rangle}\left(\boldsymbol{y}_{\langle i, j\rangle}\right)} \eta_{i \rightarrow j}\left(\boldsymbol{y}_{\langle i, j\rangle}\right) \eta_{j \rightarrow i}\left(\boldsymbol{y}_{\langle i, j\rangle}\right)=\sum_{n_{i \rightarrow j}, n_{j \rightarrow i}=0}^{\infty} \rho_{i \rightarrow j}\left(n_{i \rightarrow j}, n_{j \rightarrow i}\right) \rho_{j \rightarrow i}\left(n_{j \rightarrow i}, n_{i \rightarrow j}\right) \frac{n_{i \rightarrow j} ! n_{j \rightarrow i} !}{\left(\beta J_{\langle i, j\rangle}\right)^{n_{i \rightarrow j}+n_{j \rightarrow i}}}
$$


and

$$
\sum_{\boldsymbol{n}_{i},\left\{\boldsymbol{y}_{\langle i, j\rangle}\right\}_{j \in \partial i}} w_{i}\left(\boldsymbol{n}_{i},\left\{\boldsymbol{y}_{\langle i, j\rangle}\right\}_{j \in \partial i}\right) \prod_{j \in \partial i} \eta_{j \rightarrow i}\left(\boldsymbol{y}_{\langle i, j\rangle}\right)=\sum_{\left\{n_{i \rightarrow j}, n_{j \rightarrow i}\right\}_{j \in \partial i}} \prod_{j \in \partial i} \rho_{j \rightarrow i}\left(n_{j \rightarrow i}, n_{i \rightarrow j}\right) A_{i}\left(\sum_{j \in \partial i} n_{j \rightarrow i}, \sum_{j \in \partial i} n_{i \rightarrow j}\right)
$$

where

$$
A_{i}\left(N_{+}, N_{-}\right)=\left.\frac{1}{\beta^{N_{+}+N_{-}}} \frac{d^{N_{+}}}{d z_{+}^{N_{+}}} \frac{d^{N_{-}}}{d z_{-}^{N_{-}}} \operatorname{Tr}\left[e^{-\beta\left(V_{i}\left(a^{\dagger} a\right)-z_{+} a^{\dagger}-z_{-} a\right)}\right]\right|_{z_{+}=z_{-}=0} .
$$

As a simple illustration of this static approximation one can recover the mean-field free-energy (9) for the case of a Bethe lattice of connectivity $c$ with uniform hopping strength $J$ and the same potential energy $V(n)$ on all sites. This is indeed obtained after a short computation by taking a Poisson distribution of average $\beta J \lambda$ for the number of jumps in each direction,

$$
\rho\left(n_{+}, n_{-}\right)=e^{-2 \beta J \lambda} \frac{(\beta J \lambda)^{n_{+}+n_{-}}}{n_{+} ! n_{-} !},
$$

where $\lambda$ is proportional to the parameter $\psi$ in (9).

[1] M.P.A. Fisher, P.B. Weichman, G. Grinstein and D.S. Fisher, Phys. Rev. B 40, 546 (1989).

[2] S. Sachdev, Quantum Phase Transitions, Cambridge University Press (1999).

[3] D. Jacksh, C. Bruder, J.I. Cirac, C.W. Gardiner and P. Zoller, Phys. Rev. Lett. 81, 3108 (1998).

[4] M. Greiner, O. Mandel, T. Esslinger, T.W. Hänsch and I. Bloch, Nature 415, 39 (2002).

[5] I. Bloch, J. Dalibard and W. Zwerger, Rev. Mod. Phys. 80, 885 (2008).

[6] W. Krauth, M. Cafarel and J.-P. Bouchaud, Phys. Rev. B 45, 3137 (1992).

[7] J.K. Freericks and H. Monien, Phys. Rev. B 53, 2691 (1996).

[8] N. Teichmann, D. Hinrichs, M. Holthaus and A. Eckardt, Phys. Rev. B 79, 224515 (2009).

[9] J. K. Freericks, H. R. Krishnamurthy, Y. Kato, N. Kawashima, N. Trivedi, arXiv:0902.3435.

[10] A. P. Kampf and G. T. Zimanyi, Phys. Rev. B 47, 279 (1993).

[11] K. Sheshadri, H. R. Krishnamurthy, R. Pandit and T. V. Ramakrishnan, Europhys. Lett. 22, 257 (1993).

[12] D. van Oosten, P. Van der Straten and H. T. C. Stoof, Phys. Rev. A 63, 053601 (2001).

[13] D. Sengupta and N. Dupuis, Phys. Rev. A 71, 033629 (2005).

[14] C. Menotti and N. Trivedi, Phys. Rev. B 77, 235120 (2008).

[15] W. Krauth and N. Trivedi, Europhys. Lett. 14, 627 (1991).

[16] G.G.Batrouni and R.T.Scalettar, Phys. Rev. B 46, 9051 (1992).

[17] B. Capogrosso-Sansone, S.G. Söyler, N. Prokof'ev and B. Svistunov, Phys. Rev. A 77, 015602 (2008).

[18] B. Capogrosso-Sansone, N. Prokof'ev and B. Svistunov, Phys. Rev. B 75, 134302 (2007).

[19] B. Capogrosso-Sansone, E. Kozik, N. Prokof'ev and B. Svistunov, Phys. Rev. A 75, 013619 (2007).

[20] P. Pippan, H.G. Evertz and M. Hohenadler, arXiv:0904.1350 (2009).

[21] J.-B. Bru and T.C. Dorlas, J. Stat. Phys 113, 177 (2003).

[22] S. Adams and T. Dorlas, J. Math. Phys. 48, 103304 (2007).

[23] K. Byczuk and D. Vollhardt, Phys. Rev. B 77, 235106 (2008).

[24] A. Hubener, M. Snoek and W. Hofstetter, arXiv:0902.2212.

[25] A. Georges, G. Kotliar, W. Krauth and M.J. Rozenberg, Rev. Mod. Phys. 68, 13 (1996).

[26] A. Pelizzola, J. Phys. A: Math. Gen. 38, R309 (2005).

[27] L. Fallani, C. Fort and M. Inguscio, Adv. At. Mol. Opt. Phys. 56, 119-160 (Academic Press, 2008).

[28] R. Abou-Chacra, P.W. Anderson and D.J. Thouless, J. Phys. C : Solid State Phys. 6, 1734 (1973).

[29] M. Mézard and G. Parisi, Eur. Phys. J. B 20, 217 (2001).

[30] M. Mézard, G. Parisi and R. Zecchina, Science 297, 812 (2002).

[31] M. Mézard and A. Montanari, Information, Physics and Computation, Oxford University Press (2009).

[32] C. Laumann, A. Scardicchio and S.L. Sondhi, Phys. Rev. B 78, 134424 (2008).

[33] S. Knysh and V.N. Smelyanskiy, Phys. Rev. E 78, 061128 (2009).

[34] F. Krzakala, A. Rosso, G. Semerjian and F. Zamponi, Phys. Rev. B 78, 134428 (2008).

[35] J.W. Negele and H. Orland, Quantum Many-Particle Systems, Westview Press (1998).

[36] R. Kikuchi, Phys. Rev. 81, 988 (1951).

[37] S. Janson, T. Luczak and A. Rucinski, Random graphs, John Wiley and sons (2000).

[38] F. Kschischang, B.J. Frey and H.-A. Loeliger, IEEE Transactions on Information Theory 47, 498 (2001). 
[39] J. S. Yedidia, W. T. Freeman and Y. Weiss, in Advances in Neural Information Processing Systems 13, 689 (2001).

[40] A. Dembo and A. Montanari, arXiv:0804.4726.

[41] D. Nagaj, E. Farhi, J. Goldstone, P. Shor and I. Sylvester, Phys. Rev. B 77, 214431 (2008).

[42] M. Suzuki, Prog. Th. Phys. 56, 1454 (1976).

[43] W.F. Brinkman and T.M. Rice, Phys. Rev. B 2, 1324 (1970).

[44] U. Bissbort and W. Hofstetter, arXiv:0804.0007.

[45] K.V. Krutitsky, A. Pelster and R. Graham, New J. Phys. 8, 187 (2006).

[46] J. Friedman, A Proof of Alon's Second Eigenvalue Conjecture and Related Problems, Memoirs of the American Mathematical Society, Volume 185, Number 910 (2008).

[47] S.J. Miller, T. Novikoff and A.Sabelli, Experimental Mathematics 17, 231 (2008).

[48] A. A. Abrikosov, L. P. Gorkov and I. E. Dzyaloshinski, Methods of quantum field theory in statistical physics (Dover, 1975).

[49] F. Gerbier, S. Fölling, A. Widera, O. Mandel and I. Bloch, Phys. Rev. Lett. 96, 090401 (2006).

[50] G. Biroli, O. Parcollet and G. Kotliar, Phys. Rev. B 69, 205108 (2004).

[51] S.G. Söyler, B. Capogrosso-Sansone, N.V. Prokof'ev and B.V. Svistunov, arXiv:0811.0397 (2008).

[52] G. Thalhammer, G. Barontini, L. De Sarlo, J. Catani, F. Minardi and M. Inguscio, Phys. Rev. Lett. 100, 210402 (2008).

[53] V.W. Scarola and S. Das Sarma, Phys. Rev. Lett. 95, 033003 (2005).

[54] G. Biroli and M. Mézard, Phys. Rev. Lett. 88, 025501 (2001).

[55] G. G. Batrouni, R. T. Scalettar, G. T. Zimanyi, and A. P. Kampf, Phys. Rev. Lett. 74, 2527 (1995); S. Wessel and M. Troyer, Phys. Rev. Lett. 95, 127205 (2005); L. Dang, M. Boninsegni and L. Pollet, Phys. Rev. B 78, 132512 (2008); K. P. Schmidt, J. Dorier, A. M. Läuchli, and F. Mila, Phys. Rev. Lett. 100, 090401 (2008).

[56] L. Dang, M. Boninsegni and L. Pollet, arXiv:0812.3417

[57] E. Kim and M. H. W. Chan, Nature (London) 427, 225 (2004); E. Kim and M. H. W. Chan, Science 305, 1941 (2004).

[58] F. Gerbier, Phys. Rev. Lett. 99, 120405 (2007).

[59] A.J. Bray and M.A. Moore, J. Phys. C: Solid State Phys. 13, L655 (1980).

[60] the code of our implementation is available upon request.

[61] The initial condition should however allow for the possible spontaneous symmetry breaking $\langle a\rangle \neq 0$; we shall discuss this point further in Sec. V.

[62] Note that we compare our results for a Bethe lattice with finite connectivity with those obtained in [24] using the semicircular density of states. The latter is strictly valid in the limit of large connectivity, but this effect should be of subleading order in the large connectivity expansion.

[63] In particular the cusp in the kinetic energy at transition from the SF to the MI becomes sharper and $e_{K}$ becomes slightly smaller as we go from $\beta J=1$ to $\beta J=2$ in the first superfluid region. No further modifications are observed within our numerical errors for $\beta J>2$. 